\title{
Origin of the Different Architectures of the Jovian and Saturnian Satellite Systems
}

\author{
T. Sasaki \\ Earth and Planetary Sciences, Tokyo Institute of Technology, 2-12-1 Ookayama, Meguro-ku, \\ Tokyo 152-8551, Japan \\ takanori@geo.titech.ac.jp \\ G. R. Stewart \\ Laboratory for Atmospheric and Space Physics, University of Colorado, Campus Box 392, \\ Boulder, CO 80309-0392, USA \\ gstewart@lasp.colorado.edu \\ and \\ S. Ida \\ Earth and Planetary Sciences, Tokyo Institute of Technology, 2-12-1 Ookayama, Meguro-ku, \\ Tokyo 152-8551, Japan \\ ida@geo.titech.ac.jp
}

\begin{abstract}
The Jovian regular satellite system mainly consists of four Galilean satellites that have similar masses and are trapped in mutual mean motion resonances except for the outer satellite, Callisto. On the other hand, the Saturnian regular satellite system has only one big icy body, Titan, and a population of much smaller icy moons. We have investigated the origin of these major differences between the Jovian and Saturnian satellite systems by semi-analytically simulating the growth and orbital migration of proto-satellites in an accreting proto-satellite disk. We set up two different disk evolution/structure models that correspond to Jovian and Saturnian systems, by building upon previously developed models of an actively-supplied proto-satellite disk, the formation of gas giants, and observations of young stars. Our simulations extend previous models by including the (1) different termination timescales of gas infall onto the protosatellite disk and (2) different evolution of a cavity in the disk, between the Jovian and Saturnian systems. We have performed Monte Carlo simulations and show that in the case of the Jovian systems, four to five similar-mass satellites are likely to remain trapped in mean motion resonances. This orbital configuration is formed by type I
\end{abstract}


migration, temporal stopping of the migration near the disk inner edge, and quick truncation of gas infall caused by Jupiter opening a gap in the Solar nebula. The Saturnian systems tend to end up with one dominant body in the outer regions caused by the slower decay of gas infall associated with global depletion of the Solar nebula. The total mass and compositional zoning of the predicted Jovian and Saturnian satellite systems are consistent with the observed satellite systems.

Subject headings: planets and satellites: formation — planets and satellites: individual (Galilean satellites, Titan) — planets: rings

\section{INTRODUCTION}

The four Galilean satellites around Jupiter have similar masses and the inner three bodies are trapped in mutual mean-motion resonances. They exhibit a trend of decreasing mean bulk density with increasing orbital radius, and a diversity of axial moments of inertia (Table 1). These properties are likely caused by a progressive increase in ice-to-rock ratio with an orbital radius and different states of differentiation. The inferred undifferentiated interior of the outermost satellite, Callisto, would require a relatively long accretion timescale, $5 \times 10^{5}$ years or more (Stevenson et al. 1986; Schubert et al. 2004; Barr \& Canup 2008). On the other hand, Saturn has only one big icy satellite, Titan, which is located relatively far from Saturn. Recent Cassini data indicates that Titan also has an incompletely dierentiated interior (Iess. et al. 2010) (Table 1). The origin of the pronounced difference between the Jovian and Saturnian systems is an intriguing question.

Two important models for circum-planetary proto-satellite disks have been proposed. One is the "solids enhanced minimum mass" (SEMM) model (Mosqueira \& Estrada 2003a, 2003b; Estrada et al. 2009) and the other is an actively-supplied gaseous accretion disk (CW) model (Canup \& Ward 2002, 2006, 2009). The SEMM model postulates a massive quiescent disk with a peak surface density near $10^{5} \mathrm{~g} \mathrm{~cm}^{-2}$ and a temperature profile determined by the luminosity of the gas giant planet. The Jovian/Saturnian satellites in this model form from solid materials supplied by ablation and capture of planetesimal fragments passing through the massive disk (Mosqueira et al. 2010). The CW model postulates a low mass, viscously evolving disk with a peak surface density near 100 $\mathrm{g} \mathrm{cm}^{-2}$, that is continuously supplied by mass infall from the circum-stellar proto-planetary disk onto the circum-planetary proto-satellite disk. The disk temperature profile in the CW model is dominated by viscous heating of the evolving disk and the luminosity of the giant planet plays a lesser role. The "satellitesimals" in this model are assumed to form immediately from dust grains that are supplied by gas infall (Canup \& Ward 2006). Since the motions of the "satellitesimals" are decoupled from gas accretion, they are retained while the disk gas is actively replenished. As a result, a high dust-to-gas ratio is realized in the disk. The satellites that have grown massive enough are disposed through type I migration into the planet due to satellite-disk interaction (e.g., Ward 1986; Tanaka et al. 2002) and new generations of satellites are repeatedly accreted from the 
supplied dust grains. The finally surviving satellites are those formed at the very end of the host planet's accretion. In the present paper, we will focus on the latter model.

Canup \& Ward (2006) performed N-body simulations of accretion of satellites from small bodies and their type I migration in decaying accretion disks. They showed that the equilibrium total mass of satellites resulting from a balance between disposal by type I migration and repeated satellitesimal accretion is universally $\sim 10^{-4} M_{p}$, where $M_{p}$ is the host planet mass. The protosatellite disk gas is eventually depleted due to the decline of infall gas due to global depletion of the proto-planetary disk, then type I migration stops and satellites with total mass $\sim 10^{-4} M_{p}$ survive. The result is consistent with actual Jovian and Saturnian systems. They suggested that the difference between Jovian and Saturnian systems is caused by stochastic timing between the repeated formation/migration and depletion of disk gas.

The disk evolution in their model is regulated by the mass infall rate from the proto-planetary disk onto the proto-satellite disk (section 2.1) and the infall is responsible for the final stage gas giant planet formation. Although Canup \& Ward (2006) assumed the same proto-satellite disk evolution for both Jovian and Saturnian systems, recent theories of gas giant formation and observational data of extrasolar planets raise the possibility that the disk evolution can be different between Jovian and Saturnian systems as follows.

Unless the growth of gas giants is truncated or at least significantly slowed down at some critical mass, only a single gas giant should exist in each planetary system, because gas accretion onto a planet is a runaway process (e.g., Bodenheimer \& Pollack 1986; Pollack et al. 1996; Ikoma et al. 2000). Cores for gas giants do not generally form at the same time, and the rate of gas mass accretion in the proto-planetary disk that can supply gas to giants is observationally $\sim \mathrm{O}\left(10^{-5}\right) M_{J}$ year $^{-1}$ (where $M_{J}$ is a Jupiter mass). However, the observed data of extrasolar planets show that there are many systems with multiple gas giants. Our Solar system also has two gas giants. Furthermore, the mass distribution of extrasolar gas giants is centered at $M_{p, a v e} \sim$ a few $M_{\mathrm{J}}$ with an upper cut-off of $M_{p, \max } \sim 10 M_{\mathrm{J}}$. The observationally inferred proto-planetary disk masses have a mean value of $\sim 10 M_{J}$ and a maximum value of $\sim 100 M_{J}$ (e.g., Beckwith \& Sargent 1996). These mean and maximum values are one order greater than $M_{p, \text { ave }}$ and $M_{p, \max }$, respectively. These facts suggest that the giants did not accrete most of disk gas, so they are consistent with existence of the rapid truncation (e.g., Lin \& Papaloizou 1985) or severe decline (e.g., Lubow \& D'Angelo 2006; Tanigawa \& Ikoma 2007) of gas infall onto the planets due to gap opening in the proto-planetary disks.

Since the proposed gap opening conditions show that the critical mass for gap opening is larger in outer regions (section 2.2), outer gas giant planets, in principle, tend to be larger than inner ones. However, the outer one can be smaller, if the disk gas is depleted before the outer planet can complete its formation. Since Saturn is three times less massive than Jupiter, it is most likely that Jupiter opened up a gap to halt its growth while Saturn did not and its growth was terminated by global depletion of the proto-planetary disk (the Solar nebula). Although reduced gas infall can 
still continue after the gap opening (Lubow \& D'Angelo 2006; Tanigawa \& Ikoma 2007), the severe reduction in infall rate makes the Jovian disk evolution significantly different from the Saturnian one (see section 2).

Here, we explore a possible path to produce the pronounced different architectures of the Jovian and Saturnian satellite systems, by introducing the different evolution of proto-satellite disks due to the gap opening to the actively-supplied gaseous accretion proto-satellite disk model (Canup \& Ward 2002, 2006). The purpose of the present paper is to demonstrate how this diversity in gas giant formation can profoundly affect the regular satellite formation process.

Since we survey a wide range of parameters for initial and boundary conditions, we adopt a semi-analytical model to simulate accretion and migration of satellites from satellitesimals that has been developed by Ida \& Lin $(2004,2008)$ for modeling sequential planet formation. While the semi-analytical calculations inevitably introduce approximations, N-body simulations have to employ unrealistic initial and/or boundary conditions because of the computational limitations (e.g., Kokubo \& Ida (1998) started from planetesimals of $\sim\left(10^{-4}-10^{-3}\right) M_{\oplus}$, or Canup \& Ward (2006) replaced infalling dust grains by embryos with the isolation mass of an accreting satellite). We show in section 4.1 that our calculation produces results consistent with Canup \& Ward (2006)'s N-body simulation, although inconsistency may exist in detailed features (we need more careful comparison to reconcile the inconsistency).

In section 2, we explain our disk evolution models for Jovian and Saturnian systems in details, highlighting their differences. In section 3, semi-analytical treatments to simulate accretion of satellites from satellitesimals, orbital migration, and resonant trapping are described. The simulation results are presented in section 4 . We successfully explain the pronounced difference between Jovian and Saturnian systems in the framework of our model. We also discuss implications for the formation of Saturn's rings. Section 5 is devoted to a summary of the major results.

\section{SATELLITE FORMATION CIRCUMSTANCES}

We modify the actively-supplied disk model developed by Canup \& Ward (2002, 2006) (see section 2.1), by introducing the effects of the gap formation around a host planet's orbit. If a gap is opened, the proto-satellite disk rapidly dissipates (section 2.2), so that the mass distribution and

orbital configuration of satellites are "frozen" in the state of a relatively massive and hot disk. The hot disk condition allows retention of rocky satellites corresponding to Io and Europa. The massive disk condition could also result in formation of an inner cavity of the disk (Takata \& Stevenson 1996; also see section 2.4) that plays a crucial role in formation of resonantly trapped multiple satellites like the Galilean satellites.

Observations of Classical T-Tauri Stars (CTTSs) with relatively strong mass accretion and Weak line T-Tauri Stars (WTTSs) with relatively weak mass accretion suggest that magnetic coupling between disks and host stars becomes weak from CTTSs to WTTSs. It may imply that 
the inner cavity would vanish as disk surface density and disk accretion rate decay, corresponding to evolution from CTTS stage to WTTS one. We adopt the same picture for evolution of protosatellite disks (section 2.4).

The Saturnian disk would also have experienced the massive disk stage with an inner cavity to have resonantly trapped multiple satellites. However, if Saturn did not open up a gap in the protoplanetary disk, then as the cavity vanishes, the massive satellites are released to migrate onto the host planet. The finally surviving satellites are therefore generally formed in depleted and cold disk conditions, in which only ice-rich bodies exist (section 2.3).

\subsection{Actively-supplied disk model}

First we briefly summarize descriptions for evolution of the actively-supplied accretion disk model with relatively small mass $\sim 10^{-4} M_{p}(p=$ Jupiter, Saturn $)$ developed by Canup \& Ward (2002, 2006), which we follow in the present paper. More detailed descriptions are given in Appendix A.

Gas infall from the proto-planetary disk onto the proto-satellite disk is limited to the regions inside a critical radius $r_{c}$. Canup \& Ward (2006) assumed that the infall flux inside $r_{c}$ is independent of orbit radius and $r_{c}$ has a fixed value of $30 R_{p}$, where $R_{p}$ is the physical radius of the host planet. (Realistically, the infall mass flux can have a radial dependence and should evolve with time, but current hydrodynamic simulations of gas giant planet formation lack sufficient resolution and long enough integration times to constrain the radial and time dependence of the infall.) That is, the infall flux per unit area has a constant value of $\left(F_{p} / \pi r_{c}^{2}\right)$ at $r<r_{c}$ and vanishes at $r>r_{c}$, where $r$ is the orbital radius around the host planet and $F_{p}$ is the total mass infall rate onto the disk. In the steady accretion state of the proto-planetary disk, the infall rate is defined with a parameter $\tau_{G}$ as $F_{p, 0}=M_{p} / \tau_{G}$. Considering non-uniform inflow distributions would be an important area of future investigation.

Neglecting a diffused-out extended faint disk to make clear dynamical process of accretion and migration of proto-satellites, surface density of disk gas is given by (Appendix A)

$$
\Sigma_{g} \simeq \begin{cases}0.55 \frac{F_{p}}{3 \pi \nu} \simeq 100 f_{g}\left(\frac{M_{p}}{M_{J}}\right)\left(\frac{r}{20 R_{J}}\right)^{-3 / 4} \mathrm{~g} \mathrm{~cm}^{-2} & {\left[r<r_{c}\right]} \\ 0 & {\left[r>r_{c}\right]}\end{cases}
$$

where

$$
f_{g} \equiv\left(\frac{\alpha}{5 \times 10^{-3}}\right)^{-1}\left(\frac{\tau_{G}}{5 \times 10^{6} \mathrm{yrs}}\right)^{-3 / 4},
$$

where we used the turbulent viscosity of the proto-satellite disk with $\nu=\alpha H_{\mathrm{SD}}^{2} \Omega_{\mathrm{K}}$ (Shakura \& Sunyaev 1973), $\Omega_{\mathrm{K}}=\sqrt{G M_{p} / r^{3}}$, and temperature distribution to calculate the disk scale height $\left(H_{\mathrm{SD}}\right)$ is given by eq. (3). Since $\nu \propto T_{d} \propto F_{p}^{1 / 4}$ (eq. [3]), $\Sigma_{g} \propto F_{p} / \nu \propto F_{p}^{3 / 4} \propto \tau_{G}^{-3 / 4}$. After a 
quasi-steady state of accretion and destruction of satellites (see section 2.2 and 2.3) is established, we start the exponential decay of infall as $F_{p}=F_{p, 0} \exp \left(-t / \tau_{\text {dep }}\right)$ with $\tau_{\text {dep }}=(3-5) \times 10^{6}$ years, corresponding to global depletion of the proto-planetary disk.

We use the disk temperature in the steady state that is derived by a balance between viscous heating and black-body radiation (Appendix A),

$$
T_{d, 0} \simeq 160\left(\frac{M_{p}}{M_{J}}\right)^{1 / 2}\left(\frac{\tau_{G}}{5 \times 10^{6} \mathrm{yrs}}\right)^{-1 / 4}\left(\frac{r}{20 R_{J}}\right)^{-3 / 4} \mathrm{~K} .
$$

The disk is assumed to be vertically optically thin and isothermal (Appendix A). After the exponential decay of $F_{p}$ is imposed, the disk temperature is decreased as

$$
T_{d}=T_{d, 0} \exp \left(-\frac{t}{4 \tau_{\mathrm{dep}}}\right)
$$

The disk structure (gas surface density and temperature) is therefore regulated by $F_{p}$ (= $M_{p} / \tau_{G}$ ), in the actively-supplied disk model. We discuss how $F_{p}$ is likely to evolve with time for Jovian and Saturnian disks in the following. We will also point out that the disk inner boundary condition may also be regulated by $F_{p}$ (section 2.4 ).

\subsection{Jovian System with Gap Opening}

Except for the late stages, growth of a gas giant is a runaway process of accreting gas from the disk. The mass increase is regulated by Kelvin-Helmholtz contraction with a timescale (see e.g., Ida \& Lin 2004),

$$
\tau_{\mathrm{KH}}=\frac{M_{p}}{\dot{M}_{p}} \simeq 10^{6}\left(\frac{M_{p}}{10 M_{\oplus}}\right)^{-3} \text { years }
$$

which can have different numerical factors depending on opacity, but the runaway nature is robust. Then, $F_{p} \sim 3 \times 10^{-6}\left(M / 0.1 M_{J}\right)^{4} M_{J} / \mathrm{yr} \propto t^{4 / 3}$. After the planet accretes all the gas in feeding zone with width $\sim 2 r_{\mathrm{H}}$, where Hill radius $r_{\mathrm{H}}=\left(M_{p} / 3 M_{*}\right)^{1 / 3} r$, at $M_{p} \sim 0.3 M_{J}$ in the case of the Solar nebula, the growth would be regulated by supply of global disk accretion, $\dot{M}_{\text {disk }}$, which is $\sim 10^{-5} M_{J}$ year ${ }^{-1}$ and decays on timescales of $10^{6}-10^{7}$ years (see Fig. 1).

As we will discuss in section 2.4, satellite formation would attain a quasi-steady state between accretion of satellites from infalled materials and their migration onto the host planet. Since we are concerned with the architecture of satellite systems formed by the last survivors, we start calculations from relatively late stage with $\tau_{G}=2 \times 10^{6}$ years $\left(F_{p}=5 \times 10^{-7} M_{J}\right.$ year $\left.{ }^{-1}\right)$ for Jupiter. Although the choice of initial value of $\tau_{G}$ contains uncertainty, the critical satellite mass to start migration and disk temperature depend on $\tau_{G}$ only weakly: $M_{\text {mig }} \propto \tau_{G}^{-3 / 16}$ (eq. [15]) and $T_{d} \propto \tau_{G}^{-1 / 4}$ (eq. [3]). So, the mass distribution and compositions of final satellites do not sensitively 
depend on the initial value of $\tau_{G}$. Furthermore, since Canup \& Ward (2006)'s N-body simulations adopted similar values, we can directly compare our results with theirs.

As discussed in section 1, we assume that Jupiter opened up a gap in the Solar nebula. Then, $F_{p}$ quickly decays (eq. [9]). It is proposed that a gap is opened if both viscous and thermal conditions are satisfied (Lin \& Papaloizou 1985; Crida et al. 2006). The former condition is derived by comparison between the viscous diffusion of the gas and gravitational scattering by the planet and the critical planetary mass is given by (Lin \& Papaloizou 1985; Ida \& Lin 2004)

$$
M_{\mathrm{g}, \mathrm{vis}} \simeq \frac{40 \nu}{r_{p} \Omega_{\mathrm{K}}} M_{*} \simeq 40 \alpha\left(\frac{H_{\mathrm{PD}}}{r_{p}}\right)^{2} M_{*} \simeq 30\left(\frac{\alpha}{10^{-3}}\right)\left(\frac{r_{p}}{1 \mathrm{AU}}\right)^{1 / 2}\left(\frac{M_{*}}{M_{\odot}}\right) M_{\oplus},
$$

where $r_{p}$ is heliocentric orbital radius of the planet, $\Omega_{\mathrm{K}}$ is Keplerian frequency at $r_{p}$, and $M_{*}$ is the host star mass, For the scale height $H_{\mathrm{PD}}$ of the proto-planetary disk, we used the values of an optically thin disk (Hayashi 1981),

$$
H_{\mathrm{PD}}=0.05\left(\frac{r_{p}}{1 \mathrm{AU}}\right)^{1 / 4} r_{p}
$$

The thermal condition is determined by balance between the gravitational scattering and pressure gradient, $r_{\mathrm{H}} \sim 1.5 H_{\mathrm{PD}}$ (Lin \& Papaloizou 1985; Ida \& Lin 2004). The critical mass is given by

$$
M_{\mathrm{g}, \mathrm{th}} \simeq 400\left(\frac{r_{p}}{1 \mathrm{AU}}\right)^{3 / 4}\left(\frac{M_{*}}{M_{\odot}}\right) M_{\oplus} .
$$

Note that $M_{\mathrm{g}, \text { th }}$ is generally larger than $M_{\mathrm{g}, \mathrm{vis}}$, because typical values of the $\alpha$ parameter are $\sim 10^{-3}-10^{-2}$ for turbulence induced by magneto-rotational instability (e.g., Sano et al. 2004).

Since $M_{\mathrm{g}, \mathrm{th}}$ is comparable to Jupiter mass, it has been proposed that Jupiter's final mass is determined by the gap opening (Lin \& Papaloizou 1985; Ida \& Lin 2004). Recent fluid dynamical simulations suggest that the gap is not clear enough to halt gas accretion for $M_{p} \lesssim 5 M_{J}$ (e.g., Lubow et al. 1999; D'Angelo et al. 2003). However, the gas flow across the gap is not necessarily accreted by the planet (Dobbs-Dixon et al. 2007) and even if all the passing flow is accreted by the planet, it is only $10 \%$ of exterior disk mass accretion flow $\left(\dot{M}_{\text {disk }}\right)$ or less for $M_{p} \sim M_{J}$ and quickly vanishes with increasing $M_{p}$ (Lubow \& D'Angelo 2006; Tanigawa \& Ikoma 2007). We will show below that two orders of magnitude reduction in the mass flux onto the proto-satellite disk is enough to discriminate proto-satellite disk evolution with the gap from that without the gap.

The truncation of gas infall or its severe decline due to the gap opening depletes the circumplanetary proto-satellite disk on its own viscous diffusion timescale $\tau_{\text {diff }} \sim R_{\mathrm{SD}}^{2} / \nu$ where $R_{\mathrm{SD}}$ is the disk size and $\nu$ is disk viscosity. With the alpha model and $R_{\mathrm{SD}} \sim 20 R_{\mathrm{p}}$ (where $R_{\mathrm{p}}$ is a physical radius of the planet),

$$
\begin{aligned}
\tau_{\text {diff }} & \sim \frac{R_{\mathrm{SD}}^{2}}{\alpha H_{\mathrm{SD}}^{2} \Omega_{\mathrm{K}}} \sim\left(\frac{R_{\mathrm{SD}}}{H_{\mathrm{SD}}}\right)^{2} \frac{1}{\alpha} \frac{T_{\mathrm{K}}\left(R_{\mathrm{SD}}\right)}{2 \pi} \\
& \sim 10^{3}\left(\frac{\alpha}{10^{-3}}\right)^{-1} \text { years }
\end{aligned}
$$


where $H_{\mathrm{SD}}$ is the scale height of the proto-satellite disk and $T_{\mathrm{K}}$ is an orbital period around the planet given by

$$
T_{\mathrm{K}}(r)=0.032\left(\frac{r}{20 R_{p}}\right)^{3 / 2} \text { years. }
$$

The gap opening itself would occur on the viscous diffusion timescale for the scale height $\left(\sim H_{\mathrm{PD}}\right)$ in the proto-planetary disk, which is

$$
\tau_{\text {gap }} \sim \frac{H_{\mathrm{PD}}^{2}}{\nu} \sim\left(\frac{H_{\mathrm{PD}}}{R_{\mathrm{PD}}}\right)^{2} \frac{R_{\mathrm{PD}}^{2}}{\nu} \sim\left(10^{-3}-10^{-4}\right) \times(1-10) \mathrm{Myrs},
$$

where $R_{\mathrm{PD}}$ is size of the proto-planetary disk, which would be much larger than $5 \mathrm{AU}$, and $t_{\mathrm{dep}} \sim$ $R_{\mathrm{PD}}^{2} / \nu$ is global disk evolution timescale. Both $\tau_{\text {diff }}$ and $\tau_{\text {gap }}$ are much shorter than formation and orbital evolution timescales of satellites (see section 3.1). This separation of time scales allows us to assume that the proto-satellite disk around Jupiter was abruptly depleted when the gap opened, such that the growth and orbital migration of satellites were "frozen" at that time.

Even if we consider the imperfect truncation of infall, this feature is not changed as long as the infall rate is reduced by a factor larger than 100. The equilibrium gas surface density of the proto-satellite disk $\left(\Sigma_{g}\right)$ is determined by infall flux $\left(F_{p}\right)$ as $\Sigma_{g} \propto F_{p}^{3 / 4}$ (section 3.1). If the infall rate is decreased by two-orders of magnitude, $\Sigma_{g}$ is decreased by a factor of 30 . According to the decrease in $\Sigma_{g}$ and the associated decrease in surface density of satellitesimals, accretion and migration timescales of proto-satellites become comparable to or longer than the global depletion timescale of the proto-planetary disk ( 1-10 Myrs; Meyer et al. 2007). Therefore, although the residual infall after the gap opening is considered, the "frozen feature" is mostly preserved. We also carried out runs with the residual continuous infall with a reduction factor of 100 and confirmed the above argument. We will show that the introduction of the imperfect truncation produces results that may be rather consistent with formation of a Callisto analogue (section 4.3).

\subsection{Saturnian System without Gap Opening}

Both thermal (eq. 8) and viscous (eq. 6) conditions show that the critical mass for gap opening is larger in outer regions of the solar system. So, the final mass of Saturn should be larger than Jupiter's mass, unless significant disk mass has already been accreted by the Sun and Jupiter such that the total residual disk mass is reduced to the level of Saturnian mass when the Saturn's core is formed. Since the core accretion from planetesimals is generally slower at larger orbital radius in the proto-planetary disk (e.g., Ida \& Lin 2004), it is reasonable that Saturn's core accreted after Jupiter has fully formed (Fig. 1). Since it is most likely that Jupiter has not undergone significant type II migration, Jupiter may have formed in a partially depleted proto-planetary disk as well. Therefore, it is not unreasonable to assume that Saturn formed in a significantly depleted disk and did not open up a gap in the disk (Fig. 1). Actually, planets in further outer regions, Uranus and Neptune, have not undergone runaway gas accretion although their cores have large enough 
mass for runaway gas accretion. This timing between core accretion and disk depletion would also produce diversity of extrasolar planets (e.g., Ida \& Lin 2008).

On the assumption that Saturn did not open up a gap, the Saturnian proto-satellite disk should have been gradually depleted on the global depletion timescales of the proto-planetary disk, $t_{\text {dep }} \sim 1-10$ Myrs (Fig. 1). The many orders of magnitude difference in depletion timescales of the proto-satellite disk between Jovian $\left(\tau_{\text {diff }} \sim 10^{3}\right.$ years $)$ and Saturnian $\left(\tau_{\text {dep }} \sim 10^{6}-10^{7}\right.$ years $)$ systems would significantly affect the final configuration of satellite systems, because a typical type I migration timescale of proto-satellites $\left(\tau_{\text {mig }} \sim 10^{5}\right.$ years; Tanaka et al. 2002$)$ are in between the two timescales. Jovian satellites may retain their orbital configuration in a phase of the protosatellite disk with relatively high mass that was frozen at the time of abrupt disk depletion, while Saturnian satellites must be survivors against type I migration in the final less massive disk (Fig. 1). The migration timescale depends on the magnitude of infall flux $\left(F_{p}\right)$ through $\Sigma_{g}$ and $\nu$, which remain uncertain. However, since the migration timescale does not strongly depend on the flux $\left(\tau_{\text {mig }} \propto F_{p}^{-1 / 2}\right)$, the relation of $\tau_{\text {diff }}<\tau_{\text {mig }}<\tau_{\text {dep }}$ is not be changed by the uncertainty in $F_{p}$ even if the uncertainty is $2-3$ orders of magnitude. We start calculations from the last stage with $\tau_{G}=5 \times 10^{6}$ years $\left(F_{p}=7 \times 10^{-8} M_{J}\right.$ year $\left.{ }^{-1}\right)$ for Saturn, which is the same as the calculations by Canup \& Ward (2002, 2006).

\subsection{Difference in a Disk Inner Cavity}

Takata \& Stevenson (1996) studied magnetic coupling between the proto-satellite disk and proto-Jupiter, in order to address why Jupiter's spin rotation is substantially slower than the break-up spin rate. If Jupiter accretes disk gas rotating at Keplerian velocity near its surface, Jupiter's spin rate should be close to the break-up one. If the coupling is strong enough, angular momentum is transferred from the Jupiter's spin to the disk through the magnetic field. In this case, it is expected that the disk is truncated at the corotation radius where the disk gas corotates with the planetary spin. The critical magnetic field for the magnetic coupling is a few hundred Gauss for mass accretion rate onto a planet of $\sim 10^{-6} M_{J}$ year ${ }^{-1}$, if we use a standard formula of magnetospheric radius (Königl 1991). Such a strong magnetic field for proto-Jupiter could be consistent with a scaling argument (Stevenson 1974). Actually, Takata \& Stevenson (1996) found through numerical calculation that the proto-Jovian disk may have had an inner cavity created by the magnetic coupling.

Although evolution of the inner cavity in the proto-satellite disk is not theoretically clear, the observations for proto-planetary disks around T Tauri stars suggest that the inner cavity exists in early active stage and vanishes as the disk accretion onto a central body becomes weak. The observed spin period distribution of young stars is bimodal and CTTSs tend to rotate slower than WTTSs (Hartman 2002; Herbst \& Mundt 2005). A possible idea to account for the bimodality is as follows. The relatively high disk accretion onto CTTSs may maintain the magnetic field strong enough for the magnetic coupling to transfer spin angular momentum to the disk and create a 
cavity. On the other hand, with the lower disk accretion rate in the following WTTS stage, the coupling and hence the cavity vanishes and the host star accretes high angular momentum gas from the disk, becoming a rapid rotator (Herbst \& Mundt 2005). Recent Spitzer observations also support this idea (Rebull et al. 2006; Cieza \& Baliber 2007).

Based on the analogy of the evolution from CTTSs to WTTSs, we here assume simulation conditions of (I) a truncated boundary with an inner cavity and abrupt termination of infall for the Jovian system and (II) a non-truncated boundary without a cavity and gradual depletion of infall for the Saturnian system. The detailed settings are described in section 3.1.

The assumption may include large uncertainty and needs to be studied in greater detail. However, the purpose of the present paper is to demonstrate the important link between gas giant planet formation and satellite formation through evolution of the proto-satellite disk due to changes in infall rate and magnetic coupling.

\section{METHODS FOR SIMULATIONS FOR SATELLITE FORMATION}

Given a disk evolution model, we apply the population synthesis planet formation model (Ida \& Lin 2004, 2008) to simulate growth of proto-satellites through accretion of small bodies ("satellitesimals") and their inward orbital migration caused by tidal interactions with the gas disk.

\subsection{Evolution of infall}

In set II for Saturn, a quasi-steady state in which accretion of satellites is balanced with their removal to the host planet via type I migration is established. After the quasi-steady state is established, we start the exponential decay of infall as $F_{p}=F_{p, 0} \exp \left(-t / \tau_{\text {dep }}\right)$ with $\tau_{\text {dep }}=(3-$ $5) \times 10^{6}$ years. We integrate the systems until disk gas surface density decreases so much that satellites no longer undergo orbital migration. This setting is the same as that adopted by Canup \& Ward (2002, 2006).

In set I for Jupiter, we set the disk inner edge at a corotation radius of Jupiter $\left(\sim 2.25 R_{p}\right)$. In this case, satellites are piled up outside the disk edge. As we will argue in section 3.3, if the amount of trapped satellites exceeds a critical value, the innermost one is released. Thus, a quasi-steady state different from set II is established also in set I. After the establishment of the quasi-steady state, we start the exponential decay of infall in the same way as in set II. However, we stop integration randomly at $(0.5-0.8) \tau_{\text {dep }}$ after the exponential decay starts, which reflects the very rapid depletion of the disk due to the gap opening. We also did calculations (set I') with 100 times

reduced infall flux after the gap opening, instead of the complete termination, which corresponds to imperfect gap opening. 
While the evolution of the gas surface density of the proto-satellite disk is analytically given by eq. (1), the surface density of satellitesimals is consistently calculated with removal due to accretion by proto-satellites and supply from solid components in the infalling gas. We scale the solid surface density with a scaling factor $f_{d}$ as

$$
\Sigma_{d}=\eta_{\text {ice }} f_{d}\left(\frac{M_{p}}{M_{J}}\right)\left(\frac{r}{20 R_{J}}\right)^{-3 / 4} \mathrm{~g} \mathrm{~cm}^{-2}
$$

where $\eta_{\text {ice }}$ is an enhancement factor due to condensation of icy grains at $r>r_{\text {ice }}$ where disk temperature is lower than $160 \mathrm{~K}$; we adopt $\eta_{\text {ice }}=3$ for $r>r_{\text {ice }}$ and $\eta_{\text {ice }}=1$ otherwise. In the infall flux, the mass fraction of solid components is given by $\eta_{\text {ice }} / f$, where $f$ is the mass ratio of gas relative to silicate dust. For a fiducial value, we adopt $f=100$. If $f_{d}=f_{g}$, the gas to dust ratio of the disk is the same as that of infall. But, we found that $f_{d}$ is usually 10-100 times higher than $f_{g}$ in outer regions in a steady state (section 4.1). The method used to calculate the evolution of $\Sigma_{d}$ is described in Appendix A.

\subsection{Accretion and Migration of Proto-Satellites}

We randomly select initial locations of 10-20 satellite seeds with $M=10^{20} \mathrm{~g}\left(\sim 10^{-11} M_{J}\right.$, $\sim 10^{-10} M_{S}$ ) from a log-uniform distribution in the regions inside $r_{c}=30 R_{p}$ and calculate their growth, migration, resonant trapping, and re-generation, simultaneously calculating the evolution of $\Sigma_{d}$, until disk gas is depleted. The log-uniform distribution for initial locations corresponds to the spacing between the seeds being proportional to orbital radius, which is the simplest and natural choice (Ida \& Lin 2004). Although the choice of the initial locations is arbitrary, it would not change the overall results thanks to the effects of type-I migration. The numbers of the initial satellite seeds $(N=10-20)$ do not affect the mass and orbital distributions of final satellites as well, as long as the initial mass is small enough, because $\tau_{\text {acc }}$ is shorter for smaller $M$ (eq. 13 ) and only a few bodies undergo runaway growth from the seeds.

The accretion rate of a proto-satellite from satellitesimals is given by $\dot{M}=M / \tau_{\text {acc }}$ and the accretion timescale is (Appendix B)

$$
\begin{aligned}
\tau_{\mathrm{acc}} & \simeq 0.5\left(\frac{r}{R_{p}}\right)\left(\frac{M_{p}}{\Sigma_{d} r^{2}}\right)\left(\frac{M}{M_{p}}\right)^{1 / 3} T_{K} \\
& \simeq 10^{6} f_{d}^{-1} \eta_{\text {ice }}^{-1}\left(\frac{M}{10^{-4} M_{p}}\right)^{1 / 3}\left(\frac{M_{p}}{M_{J}}\right)^{-5 / 6}\left(\frac{r}{20 R_{J}}\right)^{5 / 4} \text { years }
\end{aligned}
$$

where $M$ is the satellite mass. The type I migration rate is $\dot{r}=r / \tau_{\text {mig }}$ and the migration timescale is (Tanaka et al. 2002)

$$
\tau_{\text {mig }} \simeq 0.3\left(\frac{c_{s}}{r \Omega_{K}}\right)^{2} \frac{M_{p}}{M} \frac{M_{p}}{r^{2} \Sigma_{g}} \Omega_{K}^{-1}
$$




$$
\begin{aligned}
& \simeq 10^{5} f_{g}^{-1}\left(\frac{M}{10^{-4} M_{p}}\right)^{-1}\left(\frac{M_{p}}{M_{J}}\right)^{-1}\left(\frac{r}{20 R_{J}}\right)^{1 / 2}\left(\frac{\tau_{G}}{5 \times 10^{6} \mathrm{yrs}}\right)^{-1 / 4} \text { years } \\
& \simeq 10^{5}\left(\frac{\alpha}{5 \times 10^{-3}}\right)\left(\frac{M}{10^{-4} M_{p}}\right)^{-1}\left(\frac{M_{p}}{M_{J}}\right)^{-1}\left(\frac{r}{20 R_{J}}\right)^{1 / 2}\left(\frac{\tau_{G}}{5 \times 10^{6} \mathrm{yrs}}\right)^{1 / 2} \text { years. }
\end{aligned}
$$

We do not include reversed torque due to a cavity (Masset et al. 2006) nor entropy gradient (Paardekooper et al. 2009) for simplicity. If a proto-satellite migrates to the host planet or collides with another proto-satellite, a next-generation seed with mass $M=10^{20} \mathrm{~g}$ is generated in the regions out of which preceding runaway bodies have migrated leaving many satellitesimals.

When $\tau_{\text {acc }}$ becomes larger than $\tau_{\text {mig }}$, migration actually starts. From eqs. (13) and (14), the critical satellite mass for the migration is given by

$$
M_{\text {mig }} \simeq 2 \times 10^{-5} \eta_{\text {ice }}^{-3 / 4}\left(\frac{M_{p}}{M_{J}}\right)^{-1 / 8}\left(\frac{r}{20 R_{J}}\right)^{-9 / 16}\left(\frac{\tau_{G}}{5 \times 10^{6} \mathrm{yrs}}\right)^{-3 / 16} M_{*} .
$$

\subsection{Trapping by Resonances and Disk Edge}

When the orbits of proto-satellites approach each other due to their differential type I migration speed, angular momentum is transferred between them through mutual gravitational perturbations and they are often trapped in mean motion resonances. As explained in Appendix C, migration in proto-satellite disks is slow enough that resonant trapping occurs with very high probability. We assume that the two approaching proto-satellites are trapped at a resonance near an orbital separation of $5 r_{\mathrm{H}}$.

For the Saturn case, we will show that large proto-satellites generally form in the outer regions and they sweep up smaller proto-satellites in the inner regions with the resonant trapping, because larger bodies migrate faster.

For the Jupiter case, migration is halted at the disk inner edge and subsequently migrating satellites are trapped in resonances one after another. Individual satellites, except the innermost one, should keep losing angular momentum through type-I migration even after the trapping. However, Ogihara \& Ida (2009) used N-body simulations that included the damping due to diskplanet interactions to produce resonantly trapped satellites that are lined up outside the inner disk edge with the innermost one pinned up at the edge even in the case without the effect of reversed type I migration torque near the edge (Masset et al. 2006). Ogihara et al. (2010) has investigated this halting mechanism in detail and found that asymmetric eccentricity damping for the body in an eccentric orbit straddling the edge transfers angular momentum from the disk to the body. Since the timescale of the eccentricity damping is much shorter than type I migration timescale by a factor of $\sim\left(c_{s} / v_{\mathrm{K}}\right)^{2} \sim \mathrm{O}\left(10^{-3}\right)$ (Tanaka et al. 2002; Tanaka \& Ward 2004), this angular momentum supply is great enough to compensate for the loss due to type I migration torques on all the trapped bodies (for details, see Ogihara et al. (2010)). For the angular momentum supply 
mechanism to work, eccentricity of the innermost body must be continuously excited. In the case of the resonantly trapped bodies, the eccentricity of the innermost body is continually excited by resonant interactions with the other bodies in the resonance. The maximum number of trapped satellites may be several, if their formula is applied.

However, there is another limitation for the trapping, which is not considered in Ogihara et al. (2010). When the total mass of the trapped satellites exceeds the total disk mass, angular momentum loss due to type I migration is absorbed by outward diffusion of the disk to outer regions in which the specific orbital angular momentum is higher. As a counter-reaction, the inner edge is no longer able to halt the satellites' inward migration. The innermost satellite is therefore released to the planetary surface. Thus, Jupiter systems also attain a quasi-steady state in which the total mass in trapped satellites is kept almost constant with small amplitude oscillations. We here adopt the latter condition rather than the condition derived by Ogihara et al. (2010). (The former condition is much more important for studying formation of close-in super-Earths in extrasolar planetary systems; Ida \& Lin (2010).)

We summarize the simulation settings for Jovian (set I) and Saturnian (set II) systems in Table II.

\subsection{Comparison with N-Body Simulations}

To check the validity of our semi-analytical model, we carried out the calculations with the same disk conditions as those in Fig. 2 of Canup \& Ward's N-body simulations (Canup \& Ward 2006). Here $F_{p}$ is constant without the exponential decay. The non-cavity condition and $f=100$ are adopted for a Jupiter mass planet. The total mass in satellites, $M_{T}$, in our simulation is plotted as a function of time for $\alpha=10^{-4}$ (solid line), $\alpha=5 \times 10^{-3}$ (dashed line), and $\alpha=5 \times 10^{-2}$ (dotted line) in Fig. 2. After an initial buildup, an equilibrium state for $M_{T}$ is attained in which disposal of proto-satellites to the host planet due to type I migration is balanced with the supply of satellitesimals from the infall. The dotted horizontal lines are Canup \& Ward (2006)'s analytical estimates for individual values of $\alpha$ with which the asymptotic values of $M_{T}$ in their N-body simulations are in good agreement.

Our semi-analytical model also reproduces evolution curves that asymptote to the dashed lines. In addition to the asymptotic values, the evolution of the initial buildup stage and oscillation frequencies in the steady state are also very similar to Canup \& Ward (2006)'s N-body simulations, although our results show more regular oscillations.

As mentioned in section 1, our semi-analytical model is complementary to N-body simulations. Because our model is faster than N-body simulations by many orders of magnitude, we can perform enough number of runs for statistical arguments and survey different boundary and initial conditions. 


\section{RESULTS OF MONTE CARLO SIMULATIONS}

For each set of simulations for Jovian and Saturnian systems (set I and set II; for details, see Table II), we repeat 100 runs with different random numbers for generating the initial locations of proto-satellite seeds and values for the parameter $\alpha$ for the disk viscosity and the timescale $\tau_{\text {dep }}$ for exponential decay of mass infall onto the disk. In addition to the initial locations of the satellite seeds, we assume log-uniform distributions for $\alpha$ in $10^{-2}-10^{-3}$ and $\tau_{\text {dep }}$ in $(3-5) \times 10^{6}$ years. The dependence on the value of $\alpha$ is also very weak as long as $\alpha$ is in this range. The inner boundary and infall truncation conditions are the most important factors that can change the results.

\subsection{Saturnian Satellite System}

We first show the results of set II, which adopts the same disk setting as Canup \& Ward (2006)'s N-body simulations. We apply this setting to simulations of Saturnian satellite systems. In this case, we found that satellites formed in outer regions of the proto-satellite disk repeatedly sweep up inner small satellites, in a steady state after an initial build up and before significant depletion of disk gas. Figure 3a shows the distributions of the number of final surviving satellites

with $M>10^{-5} M_{p}$ produced from 100 simulations for set II. Dark gray parts show the runs that produced satellite systems in which the largest satellite is mostly icy and $M>10^{-4} M_{p}$, which are analogous to the present Saturnian system.

In about $70 \%$ of the runs, only one body remains in the outer regions of the disk. Figure 4 shows the silicate-dust to gas ratio in the disk in the steady state. If $f_{d} / f_{g}=1$, the ratio is the same as that of infalling gas, $1 / 100$. This figure shows the disk is highly metal-rich in outer regions. (We are using the astronomical term "metal-rich" to denote all condensable solids, including ices, rocks and metals.) Since in the steady state, $f_{g}$ is radially constant, $f_{d}$ itself is $10-100$ times higher in outer regions than in inner regions, so that $\tau_{\text {acc }}$ is shorter in outer regions (eq. [13]). The total mass flux to satellite feeding zones is larger in the outer regions of the disk because the infall mass flux per unit area is assumed to be constant. From these two facts, large satellites are predominantly formed in the outer regions. As large satellites migrate from the outer regions, the satellites accrete satellitesimals in the inner regions during migration and trap smaller inner satellites into resonances to push them to the host planet, because type-I migration is faster for heavier satellites (Tanaka et al. 2002). This process results in significant depletion of $\Sigma_{d}$ (equivalently $f_{d}$ ) in the inner regions. In the outer regions, the retention of proto-satellites is efficient against disk gas accretion onto the host planet and the disk becomes metal-rich $\left(f_{d} / f_{g} \sim 10-100\right)$.

This process repeats until the disk gas is so depleted that type-I migration becomes ineffective. Since type-I migration is slower in the outer regions and proto-satellites keep growing near the original locations until $M$ exceeds $M_{\text {mig }}$, only one large body usually exists in outer regions after the disk gas is depleted. 
Several large bodies like the Galilean satellites are found under the same simulation setting in the previous work (Canup \& Ward 2006). Our simulations also reproduce the Galilean satellites' analogues but with a very low probability ( $\sim 5 \%$ for three bodies, $\sim 1 \%$ for four bodies). The probability we found may be much lower than the results by N-body simulations by Canup \& Ward (2006), although the evolution of the total satellite mass $M_{T}$ is very similar (Fig. 2). As mentioned in section 1, while the semi-analytical calculations inevitably introduce approximations, N-body simulations have to employ unrealistic initial and/or boundary conditions to save computational time. In the Canup \& Ward (2006)'s N-body simulations, infalling materials of dust grains are replaced by embryos with the isolation mass, which is relatively large. Since evolution of $M_{T}$ is very similar, our semi-analytical calculations and Canup \& Ward (2006)'s N-body simulations are consistent to each other to some level. To reconcile the inconsistent details, a better calibrated semi-analytical model and higher-resolution N-body simulations will be needed.

In Fig. 5a, the average mass $\left(M_{s}\right)$ and semimajor axis $(a)$ of the largest satellites with their standard deviations obtained from 67 runs that produced only one large satellite are plotted and compared with Titan (open circle). The mass and semimajor axis of the largest bodies agree with those of Titan within $1 \sigma$ standard deviations of 67 runs. Furthermore, the largest body contains (1) more than $95 \%$ of the total satellite system mass in 31 runs out of 67 cases, (2) more than $90 \%$ in 21 runs, and (3) less than $90 \%$ in 15 runs. The actual significant mass concentration in Titan ( $\sim 95 \%$ of the Saturnian satellite system mass) is reproduced in our simulations.

The colors of the results in Fig. 5 show the average fraction of rocky (dark gray) and icy (light gray) components. Since the final satellites are the last survivors formed in the last stage with low disk temperatures due to reduced viscous heating, they are generally composed of ice. These satellites were usually formed on long timescales $\sim \mathrm{O}\left(10^{6}\right)$ years, which suggests that Titan is not fully differentiated. In fact, recent Cassini data indicated that Titan has an inertia factor of 0.34 and is therefore incompletely differentiated just like Callisto (Iess. et al. 2010).

Therefore, our calculations are able to produce many features (orbits, masses, and compositions) of Saturnian satellite system. The results of typical successful runs are shown in Fig. 6a. In these cases, we produced only one large satellite at Titan's location and some smaller satellites at inner satellites' region.

Castillo-Rogez et al. (2007, 2009) have argued that Saturn's outer regular satellite, Iapetus, must have formed between 3.4 and 5.4 Myrs after the production of the calcium-aluminum inclusions (CAIs) found in meteorites in order to accrete the necessary amount of the short-lived radioisotope ${ }^{26} \mathrm{Al}$ to ensure its partial differentiation and tidal despinning to its current state of synchronous rotation. Mosqueira et al. (2010) also emphasize the important constraint that Iapetus places on any successful model of Saturnian satellite formation. In future work we plan to address the formation of Iapetus by considering a more detailed evolution model of the outer edge of the proto-satellite disk. 


\subsection{Jovian Satellite System}

For the Jovian case (set I), our numerical simulations produced 4 or 5 large bodies with $M>10^{-5} M_{p}$ in about $80 \%$ of the runs (Fig. 3b). Dark gray parts show the runs that the produced satellite systems in which the inner two bodies are rocky and the outer two are icy satellites. In the four large satellite cases, the inner three are trapped in mutual resonances in all runs, while the outermost one is not trapped in a resonance in about half of the runs. Such resonant trapping of inwardly migrating satellites would reproduce the detailed orbital properties of the Galilean satellites (Peale \& Lee 2002). Note that in the Saturnian system, this stage may be followed by the low- $\Sigma_{g}$ phase without a cavity, in which all the trapped satellites fall onto the host planet and only one dominant icy body formed in the last stage remains, as explained in section 4.1.

Figure $3 \mathrm{~b}$ shows that for set I, formation of four large satellites is the most likely outcome. In Fig. 5b, their averaged mass and semimajor axis and their standard deviations from 39 runs that produced four large satellites are plotted with Galilean satellites (open circles). The simulated masses and orbits are consistent with those of Galilean satellites.

In Fig. 5b, the compositions of the simulated satellites are also indicated. In more than half of the runs, the inner two bodies are composed mostly of rocky materials and while the outer two are formed mostly of icy materials that have migrated from the regions outside the ice line in the protosatellite disk. In the Jovian systems, the final satellites are those formed in disks with relatively high infall rates onto the disk, the disk temperature is high enough ( $T_{d} \propto F_{p}^{1 / 4}$; eq. [A2]) that rocky materials are a major component of satellites formed in the inner regions (eq. [3]). Outer satellites are formed in the outer regions and migrate inward to be trapped in resonances. Our simulations are consistent with the compositional gradient observed in the Galilean satellites. The results of typical successful runs are shown in Fig. 6b. In these cases, we produced four large satellites those correspond to Galilean satellites for both sizes and locations. Although some smaller satellites were also formed near Io's location, they could be merged with Io afterward.

The moment of inertia of Callisto suggests that its interior is differentiated only partially. It implies that Callisto must be formed slowly such as accretion timescale $5 \times 10^{5}$ years or more (Stevenson et al. 1986; Schubert et al. 2004; Barr \& Canup 2008). In the case of Jovian system, the outermost large satellite corresponding to Callisto is formed on timescales of $\mathrm{O}\left(10^{5}\right)$ years in some cases, but generally the timescales would be much shorter than the above estimate.

We also performed additional set (set I') of calculations with the effects of imperfect gap opening, that is, after the gap opening, we do not truncate the infall but reduce the infall rate by a factor of 100. In almost all runs, we found that after the gap opening one additional big body tends to accrete without migration in outer regions of the decayed disk on timescales longer than $\mathrm{O}\left(10^{6}\right)$ years, which comes from the fact that $\tau_{\text {acc }} \propto f_{d}^{-1}$. Because the outermost body hardly migrates, it is not captured by a resonance. The pronounced difference between Jovian and Saturnian satellite systems is preserved, although the most probable number of remaining satellites is five. Thus, if we take into account the effects of imperfect gap opening, we found that the formation timescale and 
orbital configuration of the outermost satellite in our simulations becomes much more consistent with Callisto. To discuss details of final orbital configurations and formation timescale of the outermost satellite, we need to take into account the time evolution of radial dependence of gas and dust infall rates.

\subsection{Formation of Saturn's Rings}

We will comment on another pronounced difference between Jovian and Saturnian systems, planetary rings. The discovery by the Cassini mission that most of the mass in Saturn's rings is organized into opaque, elongated clumps of ring particles (Colwell et al. 2007, 2009; Hedman et al. 2007) has led to revised estimates of the total mass contained in the rings. By comparing the amount of light transmitted by the rings during stellar occultations with the transparency of local N-body simulations of the rings, Robbins et al. (2010) estimate that the total mass of Saturn's rings may be twice the mass of the satellite, Mimas, or about $10^{-7} M_{p}$. The larger mass estimate for Saturn's main rings makes a ring formation scenario that captures a passing large Kuiper belt object less probable and also causes problems for ring formation by the collisional breakup of a former Saturnian satellite unless the impact occurred within the first 750 Myrs after Saturn's formation when the impactor flux at Saturn's orbit was much higher than today (Charnoz et al. 2009). Spectroscopic observations indicate that Saturn's rings are mainly composed of water ice with less than a few percent by mass of a non-icy component (Cuzzi et al. 2009). This is a much smaller fraction of rock than is typical for the icy regular satellites in the Saturn system, which presents a problem for ring formation by the breakup of a former satellite unless substantial amounts of silicate rock are hidden under ice regoliths in the dense core of the B ring. Recent Cassini observations indicate the existence of macroscopic bodies that produce "propeller"-shaped structures, in the A ring. The existence of these macroscopic bodies gives new support to a scenario of the ring origin through the catastrophic disruption of a massive progenitor, implying that the ring is old (Tiscareno et al. 2008). The origin of Saturn's main ring system is therefore still an unsolved question.

Charnoz et al. (2009) showed that the ring may be formed from an ancient satellite which was originally in Saturn's Roche zone (RZ) and was destroyed by a passing comet. (The small satellite itself may have formed outside RZ and migrated or been scattered into RZ.) In this model, a key aspect is the survival of the satellite against tidal evolution in the planet's RZ. Since the tidal bulge of the host planet leads to inward migration if the satellite's orbital radius $(r)$ is below the synchronous radius $\left(R_{\text {sync }}\right)$ with the planet's spin. Typical inward migration timescales are $\sim$ $\mathrm{O}\left(10^{8}\right)$ years for $M \sim 10^{-7} M_{p}$, which is much shorter than the Solar system age, so that for the satellite to survive, the condition $R_{\text {sync }}<r<R_{\mathrm{RZ}}$ is required (Charnoz et al. 2009), where $R_{\mathrm{RZ}}$ is the radius of RZ and is inversely proportional to cube root of the material density of the satellite $\left(R_{\mathrm{RZ}} \propto \rho_{s}^{-1 / 3}\right)$. For current Jupiter, Saturn, Uranus and Neptune, $R_{\text {sync }} / R_{p}=2.24,1.86,3.22$ and 3.36 , respectively. Even if a satellite is an icy body with material density of $\rho_{s}=1 \mathrm{~g} \mathrm{~cm}^{-3}, R_{\mathrm{RZ}}$ for 
Uranus and Neptune are $2.79 R_{p}$ and $2.89 R_{p}$ that are still smaller than their $R_{\text {sync }}$. Thus, Uranus and Neptune may be unable to maintain satellites inside their RZs (Charnoz et al. 2009).

Our results indicate that the inner satellite, which can be the seed of the ring, should be mixture of ice and rock for Saturnian system, while it may be rocky for Jovian system (Fig. 5). Since rocky bodies have larger values of $\rho_{s}, R_{\mathrm{RZ}}$ may be smaller for Jupiter. For example, $R_{\mathrm{RZ}}=1.90 R_{p}$ with $\rho_{s}=1.5 \mathrm{~g} \mathrm{~cm}^{-3}$ for Saturn, which is larger than Saturn's $R_{\text {sync }}=1.86 R_{p}$, while $R_{\mathrm{RZ}}=1.99 R_{p}$ with

$\rho_{s}=2.5 \mathrm{~g} \mathrm{~cm}^{-3}$ for Jupiter, which is smaller than Jupiter's $R_{\text {sync }}=2.24 R_{p}$. Therefore, Saturn is the most plausible planet to maintain a satellite inside the RZ against the tidal evolution. If the disruption scenario is correct, our result that finally surviving satellites are formed in a warmer disk for Jovian system than for Saturnian system is consistent with the fact that Saturn has a massive ring while Jupiter does not.

\section{CONCLUSION}

We simulated growth and orbital migration of proto-satellites in an accreting circum-planetary disk that is modified from the disk model by Canup \& Ward $(2002,2006)$, in order to address the different architectures between Jovian and Saturnian satellite systems:

1. Jovian system has four similar-sized satellites (Galilean satellites) locked in mean-motion resonances except the outermost one (Callisto), while Saturnian system has only one big satellite, Titan, in outer region.

2. Inner two satellites are rocky and outer two are mostly icy for Jovian system, while Titan is mostly icy.

We modified the semi-analytical model for the population synthesis model for planet formation developed by (Ida \& Lin 2004, 2008) to apply the model for satellite formation, introducing the effect of resonant trapping that characterizes Galilean satellites. The semi-analytical model reproduces the results consistent with N-body simulations by Canup \& Ward (2006), although details such as mass distribution of surviving satellites are not necessarily consistent (it is a future work to reconcile the inconsistency).

We considered a coupled system of gas giant planet formation and satellite formation around the planet, although planet formation is not simultaneously simulated. The fact that multiple gas giant systems are found in extrasolar systems in addition to our Solar system suggests that gas accretion onto gas giants is truncated or at least significantly reduced by gap formation in the proto-planetary disk. Since Saturn is smaller than Jupiter and it is theoretically expected that Saturnian core is formed later than that of Jovian core, we consider a working hypothesis that Jupiter opened up a gap while Saturn did not. Accordingly, we assume the followings:

1. Gas infall onto a proto-satellite disk is truncated or significantly reduced at the time of gap 
opening for Jupiter while that onto Saturnian disk slowly decays as the proto-planetary disk (the Solar nebula) globally decayed on a timescale of $\tau_{\text {dep }} \sim 1-10$ Myrs. The Jovian disk decays on its viscous diffusion timescale of $\tau_{\text {diff }} \sim 10^{3}$ years after the gap opening.

2. Based on inference from observations of different spin periods between CTTSs and WTTSs, the proto-satellite disk has an inner cavity in the early, high disk accretion stage and the cavity vanishes in the subsequent, low disk accretion stage.

Since typical type I migration timescales are much longer than $\tau_{\text {diff }}$ and much shorter than $\tau_{\text {dep }}$, the Jovian satellite system may have been "frozen" at the time of gap opening in which disk mass and temperature were relatively high while the Saturnian satellites may be final survivors in the last stage in which disk mass and temperature were relatively low. Thus, we carried out simulations with a cavity and abrupt decay of disk gas for Jupiter and those without a cavity and with slow disk depletion on timescales of 1-10 Myrs for Saturn. Then, we have found the following results:

1. In Jupiter-condition systems, four or five similar-sized satellites are formed in $80 \%$ of the runs and the inner ones tend to be locked in mean-motion resonances due to type I migration and its stoppage at the disk inner edge. In Saturn-condition systems, predominant bodies repeatedly accrete in outer regions and migrate onto the surface of the host planet, sweeping up inner small satellites. In $70 \%$ of runs, only one big body remains. The big bodies have more than $95 \%$ of total mass of remaining bodies in the system in about half of the runs.

2. Because Jupiter-condition systems are formed in relatively hot disks, the inner two satellites tend to be rocky and outer ones are ice-rich. On the other hand, in Saturn-condition systems, the finally surviving bodies form in the longer lasting cold disks and are therefore ice-rich. Moreover, the surviving massive bodies coresponding to Titan were usually formed on long timescales ( $\sim$ Myrs), which is consistent with the incomplete differentiation of Titan's interior.

If we consider a small amount of residual mass infall onto the circum-Jovian disk after the gap opening, the formation timescale of the outermost satellite is longer than Myrs, which is consistent with incomplete differentiation of Callisto's interior. Thus, our simulations produced the Galilean satellites' analogues and the Titan's analogues in each setting with high probability.

We have demonstrated that our assumption on gap formation and associated existence of a disk cavity naturally explain the different architectures between Jovian and Saturnian satellite systems, although more detailed fluid dynamical simulations are needed for the radial dependence and its time evolution of infall onto proto-satellite disks and gap formation in proto-planetary disks, and more detailed MHD simulations are necessary for evolution of a cavity. The different architectures may be fossil evidence that Jupiter opened up a clear gap in the proto-planetary disk to terminate its growth. This result gives deep insights into the mass distribution and multiplicity of extrasolar gas giant planets (Ida \& Lin 2008) as well as into the observed bimodal distributions of spin periods of young stars (Herbst \& Mundt 2005). 
Notice that the Jovian/Saturnian satellite systems are analogous to extrasolar super-Earth systems, because super-Earths accrete only solid materials and have masses of $\sim\left(10^{-5}-10^{-4}\right) M_{\odot}$. A high fraction of solar-type stars may have close-in super-Earths (e.g., Mayor et al. 2009), while many systems including the Solar system do not have any close-in super-Earths. Our results should also give deep insights into the origin of the diversity of super-Earth systems found around other stars.

We gratefully acknowledghe the referee's helpful comments. This work was supported by a JSPS Research Fellowship. G. R. Stewart was supported by NASA's Outer Planet Research Program and thanks the Tokyo Institute of Technology for hosting his visit in 2007. S. Ida thanks the University of Colorado at Boulder for hosting his visit in 2008.

\section{A. CIRCUM-PLANETARY DISK MODEL}

The growth rate of a proto-satellite depends on the surface density of solid materials of the proto-satellite disk (Ida \& Lin 2004) and its orbital migration rate depends on the surface density of gas (Tanaka et al. 2002). We adopt the model of an actively-supplied accretion disk by Canup \& Ward $(2002,2006)$. The asymptotic disk gas surface density distribution at $r<r_{c}$, in which the gas from the circum-stellar proto-planetary disk inflows, is given by

$$
\Sigma_{g}=\frac{F_{p}}{3 \pi \nu}\left[1-\frac{4}{5} \sqrt{\frac{r_{c}}{r_{d}}}-\frac{1}{5}\left(\frac{r}{r_{c}}\right)^{2}\right] \simeq 0.55 \frac{F_{p}}{3 \pi \nu},
$$

where $F_{p}$ is the total infall rate to whole disk regions at $r<r_{c}, \nu$ is disk gas viscosity, and $r_{d}$ is the diffused-out disk outer edge, which is $\sim 150 R_{p}$. The critical radius for the infall, $r_{c}$, is defined as the orbital radius at which the specific angular momentum of a circular orbit equals that of the inflow average. We adopt $r_{c}=30 R_{p}$ that is consistent with three-dimensional hydrodynamic simulations with high spatial resolution within the planet's Hill sphere (Machida et al. 2008, Machida 2009).

The total infall rate is defined with a parameter $\tau_{G}$ as $F_{p, 0}=M_{p} / \tau_{G}$ for the steady accretion state. According to Canup \& Ward (2006), we adopt $\tau_{G}=5 \times 10^{6}$ years for Saturn while $2 \times 10^{6}$ years for Jupiter. The infall rate decays exponentially with timescale $\tau_{\text {dep }}=3 \times 10^{6}-5 \times 10^{6}$ years in the final state of the Saturnian case and abruptly vanishes due to the gap opening in the proto-planetary disk for the Jovian case. The second and third terms in the bracket of the above equation are correction terms due to outward diffusion. If they are neglected, $\Sigma_{g}$ is no other than the distribution of an ordinary steady accretion disk.

The proto-satellite disk is heated by luminosity from the central planet, viscous dissipation, and energy dissipation associated with the difference between the free-fall energy of the incoming gas and that of a Keplerian orbit. We make the simplification that the viscous dissipation is the dominant energy source, as is typically the case in most of the regular satellite regions (Canup \& 
Ward 2002). Then, the photosurface temperature of the proto-satellite disk $\left(T_{d}\right)$ is determined by a balance between viscous heating and blackbody radiation from the photosurface,

$$
\sigma_{S B} T_{d}^{4} \simeq \frac{9}{8} \Omega_{K}^{2} \nu \Sigma_{g} \simeq \frac{0.55 \times 3}{8 \pi} \Omega_{K}^{2} F_{p}
$$

where $\Omega_{K}$ is Keplerian frequency. That is, in the steady state with a constant $F_{p, 0}$,

$$
T_{d, 0} \simeq 160\left(\frac{M_{p}}{M_{J}}\right)^{1 / 2}\left(\frac{\tau_{G}}{5 \times 10^{6} \mathrm{yrs}}\right)^{-1 / 4}\left(\frac{r}{20 R_{J}}\right)^{-3 / 4} \mathrm{~K} .
$$

After the exponential decay of $F_{p}$ is imposed, the disk temperature decreases as

$$
T_{d}=T_{d, 0} \exp \left(-\frac{t}{4 \tau_{\mathrm{dep}}}\right)
$$

Note that $T_{d}$ depends solely on $F_{p}$ (equivalently, $\tau_{G}$ ) but is independent of the value of the viscosity $\nu$ (equivalently, $\alpha$ ) which has a large uncertainty. After establishment of a steady state, $T_{d}$ is decreased from this value according to the decay of $F_{p}$, so proto-satellites are mostly composed of ice in the final state.

The midplane temperature, $T_{c}$, is given by $T_{c} \simeq(1+3 \tau / 8)^{1 / 4} T_{d}$, where $\tau$ is optical depth. Because we are concerned with disk temperature mainly for evolution of the ice line at $T_{d} \sim 160$ $\mathrm{K}$ that divides compositions of satellitesimals and is located in relatively outer regions, we assume that $T_{c} \simeq T_{d}$ to avoid uncertainty in opacity.

Adopting the alpha prescription for viscosity (Shakura \& Sunyaev 1973), $\nu \simeq \alpha c_{s}^{2} / \Omega_{K}$, the disk gas surface density distribution becomes

$$
\Sigma_{g} \simeq 100 f_{g}\left(\frac{M_{p}}{M_{J}}\right)\left(\frac{r}{20 R_{J}}\right)^{-3 / 4} \mathrm{~g} \mathrm{~cm}^{-2}
$$

where

$$
f_{g} \equiv\left(\frac{\alpha}{5 \times 10^{-3}}\right)^{-1}\left(\frac{\tau_{G}}{5 \times 10^{6} \mathrm{yrs}}\right)^{-3 / 4}
$$

Since $\nu \propto T_{d} \propto F_{p}^{1 / 4}, \Sigma_{g} \propto F_{p} / \nu \propto F_{p}^{3 / 4} \propto \tau_{G}^{-3 / 4}$.

For simplicity, it is assumed (Canup \& Ward 2002, 2006) that the infall flux per unit area is constant $\left(F_{p} / \pi r_{c}^{2}\right)$ at $r<r_{c}$ and vanishes at $r>r_{c}$. The total disk mass inside $r_{d}$ is

$$
M_{d i s k}=\int_{0}^{r_{d}} \Sigma_{g} 2 \pi r d r \simeq 1 \times 10^{-4} f_{g}\left(\frac{M_{p}}{M_{J}}\right)\left(\frac{r_{d}}{150 R_{J}}\right)^{5 / 4} M_{J}
$$

We scale the solid surface density with a scaling factor $f_{d}$ as

$$
\Sigma_{d}=\eta_{\text {ice }} f_{d}\left(\frac{M_{p}}{M_{J}}\right)\left(\frac{r}{20 R_{J}}\right)^{-3 / 4} \mathrm{~g} \mathrm{~cm}^{-2}
$$


where $\eta_{\text {ice }}$ is an enhancement factor due to condensation of icy grains at $r>r_{\text {ice }}$ where disk temperature is lower than $160 \mathrm{~K}$; we adopt $\eta_{\text {ice }}=3$ for $r>r_{\text {ice }}$ and $\eta_{\text {ice }}=1$ otherwise. Since the satellitesimals' motions are decoupled from gas accretion/diffusion, they stay on-site and $\Sigma_{d}$ remains zero for $r>r_{c}$. The infall rate of solid components per unit area of the disk is given by $\left(F_{p} / \pi r_{c}^{2}\right) /\left(f / \eta_{\text {ice }}\right)$, where $f$ is the ratio of gas to rocky dust grains in the solar nebula. In our calculations, the radial distribution of solid materials $\left(\Sigma_{d}\right.$ or $\left.f_{d}\right)$ in the proto-satellite disk quickly relaxes to a steady state in which the supply of solid components in the infalling gas is balanced with the removal due to accretion by proto-satellites and their migration. In the steady state, the rate of increase of $f_{d}$ due to infall is

$$
\begin{aligned}
\frac{d f_{d}}{d t} & =\frac{d \Sigma_{d} / d t}{\Sigma_{d}\left(f_{d}=1\right)}=\frac{\left(\eta_{\text {ice }} / f\right)\left(F_{p} / \pi r_{c}^{2}\right)}{\Sigma_{d}\left(f_{d}=1\right)} \\
& =0.029\left(\frac{M_{p}}{M_{J}}\right)^{-2 / 3}\left(\frac{f}{100}\right)^{-1}\left(\frac{\tau_{G}}{5 \times 10^{6} \mathrm{yrs}}\right)^{-1}\left(\frac{r}{20 R_{J}}\right)^{3 / 4} \text { year }^{-1} .
\end{aligned}
$$

The increase in the masses of the proto-satellites is subtracted from their feeding zones of width $10 r_{\mathrm{H}} \cdot f_{d}$ is assumed to be locally uniform in the feeding zones. The proto-satellites migrate inward and eventually fall onto the host planets in the presence of disk gas. Thereby, the total amount of solid materials is decreased by the growth and migration of proto-satellites. Our calculation shows that asymptotic values of $f_{d}$ are $\sim 1$ in inner regions and $(10-100) f_{g}$ in outer regions (Fig. 4),

which means that the outer disks are generally metal-rich due to the higher retention rate of solid materials compared to the gas.

\section{B. ACCRETION AND MIGRATION RATES OF PROTO-SATELLITES}

The growth and migration of satellites in circum-planetary proto-satellite disks proceed similarly to those of solid planets in circum-stellar proto-planetary disks that have been studied in detail (Ida \& Lin 2004). Given a disk model, the accretion and migration rates of proto-satellites are analytically evaluated. For values of velocity dispersion $(\sigma)$ larger than the Hill velocity $\left(r_{\mathrm{H}} \Omega_{K}\right)$ but smaller than surface escape velocity from satellites, the accretion rate of a proto-satellite with mass $M$ at an orbital radius $r$ is (Ida \& Lin 2004)

$$
\begin{aligned}
\dot{M} & \simeq C \pi R^{2} \rho_{d}\left(\frac{2 G M}{R \sigma^{2}}\right) \sigma \simeq 2 C \pi R^{2} \Sigma_{d} \Omega_{K}\left(\frac{G M}{R \sigma^{2}}\right) \\
& \simeq 2 \pi C\left(\frac{R}{r}\right)\left(\frac{\Sigma_{d} r^{2}}{M_{p}}\right)\left(\frac{r \Omega_{K}}{\sigma}\right)^{2} M \Omega_{K},
\end{aligned}
$$

where $\rho_{d}$ is the spatial mass density of solid components $\left(\rho_{d} \simeq \Sigma_{d} \Omega_{K} / \sigma\right), R$ is a physical radius of the satellite, and $C$ is a numerical factor of 2-3 (Stewart \& Ida 2000; Ohtsuki et al. 2002). In principle, the velocity dispersion $\sigma$ of satellitesimals would be determined by a balance between gas drag damping and stirring by the satellite. However, because the typical mass of satellitesimals, 
which determines gas drag damping, accreted by the satellite is not clear, we simply set

$$
\frac{\sigma}{r \Omega_{K}} \simeq \beta\left(\frac{M}{3 M_{p}}\right)^{1 / 3}
$$

with $\beta \simeq 10$, following the case of planetesimals in the proto-planetary disks (Ida \& Lin 2004). The accretion timescale is

$$
\begin{aligned}
\tau_{\text {acc }} & =\frac{M}{\dot{M}} \simeq 0.5\left(\frac{r}{R_{p}}\right)\left(\frac{\rho}{\rho_{p}}\right)^{-1 / 3}\left(\frac{M_{p}}{\Sigma_{d} r^{2}}\right)\left(\frac{M}{M_{p}}\right)^{1 / 3}\left(\frac{\beta}{10}\right)^{2} T_{K} \\
& \simeq 10^{6} f_{d}^{-1} \eta_{\text {ice }}^{-1}\left(\frac{\rho}{\rho_{p}}\right)^{-1 / 3}\left(\frac{M}{10^{-4} M_{p}}\right)^{1 / 3}\left(\frac{M_{p}}{M_{J}}\right)^{-5 / 6}\left(\frac{\beta}{10}\right)^{2}\left(\frac{r}{20 R_{J}}\right)^{5 / 4} \text { years, }
\end{aligned}
$$

where we used $C=2.5$ and $\rho$ and $\rho_{p}$ are bulk densities of the satellite and the host planet (we neglect the dependence on $\left.\rho / \rho_{p}\right)$.

The type I migration timescale (Tanaka et al. 2002) is

$$
\begin{aligned}
\tau_{\text {mig }} & =\frac{r}{\dot{r}}=\frac{1}{2.7+1.1 q_{g}}\left(\frac{c_{s}}{r \Omega_{K}}\right)^{2} \frac{M_{p}}{M} \frac{M_{p}}{r^{2} \Sigma_{g}} \Omega_{K}^{-1} \\
& \simeq 10^{5} \frac{1}{f_{g}}\left(\frac{M}{10^{-4} M_{p}}\right)^{-1}\left(\frac{M_{p}}{M_{J}}\right)^{-1}\left(\frac{r}{20 R_{J}}\right)^{1 / 2}\left(\frac{\tau_{G}}{5 \times 10^{6} \mathrm{yrs}}\right)^{-1 / 4} \text { years },
\end{aligned}
$$

where $q_{g}$ is defined by $q_{g}=-\ln \Sigma_{g} / \ln r$.

\section{RESONANT TRAPPING}

For two satellites on nearly circular orbits, the expansion of the difference in their semimajor axes $(b)$ during an encounter is given by linear calculations (Goldreich \& Tremaine 1982; Hasegawa \& Nakazawa 1990) as $\delta b \simeq 30\left(b / r_{\mathrm{H}}\right)^{-5} r_{\mathrm{H}}$. The Hill radius $\left(r_{\mathrm{H}}\right)$ is defined by $r_{\mathrm{H}}=\left(M / 3 M_{p}\right)^{1 / 3} r$, where $M, M_{p}$ and $r$ are masses of the proto-satellite and the central planet, and the orbital radius of the proto-satellite, respectively. Since the scattering occurs at every synodic period $\left[T_{\mathrm{syn}}=2 \pi r /((d \Omega / d r) b) \simeq\left(4 \pi r / 3 b \Omega_{\mathrm{K}}\right)\right]$

$$
\frac{d b}{d t} \simeq \frac{\delta b}{T_{\mathrm{syn}}} \simeq 7\left(\frac{b}{r_{\mathrm{H}}}\right)^{-4}\left(\frac{r_{\mathrm{H}}}{r}\right)^{2} v_{\mathrm{K}} .
$$

The embryos may be trapped in a resonance close to the equilibrium value of $b$ that satisfies

$d b / d t=v_{\text {mig }}$, where $v_{\text {mig }}$ is relative radial migration speed for the non-interacting case that is given by equation (14). Then, the equilibrium value is given by

$$
b_{\text {trap }} \simeq 0.29\left(\frac{M}{10^{-4} M_{J}}\right)^{1 / 6}\left(\frac{v_{\mathrm{mig}}}{v_{\mathrm{K}}}\right)^{-1 / 4} r_{\mathrm{H}} .
$$


If the estimated value of $b_{\text {trap }}$ is smaller than $2 \sqrt{3} r_{\mathrm{H}}$, the trapping does not actually occur, because the separation is within their feeding zone and $\delta b$ saturates due to the non-linear effect (e.g., Ida 1990). Substituting eq. (14) into eq. (C2), we find that

$$
b_{\text {trap }} \simeq 16 f_{g}^{-1 / 4}\left(\frac{M}{10^{-4} M_{J}}\right)^{-1 / 12}\left(\frac{M_{p}}{M_{J}}\right)^{-1 / 4}\left(\frac{r}{20 R_{J}}\right)^{1 / 8}\left(\frac{\tau_{G}}{5 \times 10^{6} \text { year }}\right)^{-1 / 16} r_{\mathrm{H}} .
$$

Since the estimated value of $b_{\text {trap }}$ is much greater than $2 \sqrt{3} r_{\mathrm{H}}$, we assume perfect trapping in our simulations. In our simulations, several satellites are usually trapped, so the final trapping location tends to be deeper than the above value due to collective interactions, which is also shown in N-body simulations (e.g., Ogihara \& Ida 2009). We therefore assume that the two approaching proto-satellites are trapped at a resonance near $b=5 r_{\mathrm{H}}$. 


\section{REFERENCES}

Barr, A. C., \& Canup, R. M. 2008, Icarus, 198, 163

Beckwith, S. V. W., \& Sargent, A. I. 1996, Nature, 383, 139

Bodenheimer, P., \& Pollack, J. B. 1986, Icarus, 67, 391

Canup, R. M., \& Ward, W. R. 2002, AJ, 124, 3404

Canup, R. M., \& Ward, W. R. 2006, Nature, 441, 834

Canup, R. M., \& Ward, W. R. 2009, in Europa, ed. R. T. Pappalardo, W. B. McKinnon, \& K. Khurana (Tucson: Univ. Arizona Press), 59

Castillo-Rogez, J. C., Matson, D. L., Sotin, C., Johnson, T. V., Lunine, J. I., \& Thomas, P. C. 2002, Icarus, 190, 179

Castillo-Rogez, J. C., Johnson, T. V., Lee, M. H., Turner, N. J., Matson, D. L., \& Lunine, J. I. 2009, Icarus, 204, 658

Cieza, L., \& Baliber,N. 2007, ApJ, 671, 605

Charnoz, S., Morbidelli, A., Dones, L., \& Salmon, J. 2009, Icarus, 199, 413

Colwell, J. E., Esposito, L. W., Sremcevic, M., Stewart, G. R., \& McClintock, W. E. 2007, Icarus, 190,127

Colwell, J. E., Nicholson, P. D., Tiscareno, M. S., Murray, C. D., French, R. G., \& Marouf, E. 2009, in Saturn from Cassini-Huygens, ed. M. K. Dougherty, L. W. Esposito, \& S. M. Krimigis (New York: Springer), 375

Cuzzi, J., Clark, R., Filacchione, G., French, R., Johnson, R., Marouf, E., \& Spilker, L. 2009, in Saturn from Cassini-Huygens, ed. M. K. Dougherty, L. W. Esposito,\& S. M. Krimigis (New York: Springer), 459.

Crida, A., Morbidelli, A., \& Masset, F. 2006, Icarus, 181, 587

D’Angelo, G., Kley, W., \& Henning, T. H. 2003, ApJ, 586, 540

Dobbs-Dixon, I., Li, S. L., \& Lin, D. N. C. 2007, ApJ, 660, 791

Estrada, P. R., Mosqueira, I., Lissauer, J. J., D’Angelo, G., \& Cruikshank, D. P. 2009, in Europa, ed. R. T. Pappalardo, W. B. McKinnon, \& K. Khurana (Tucson: Univ. Arizona Press), 27

Goldreich, P., \& Tremaine, S. 1982, ARA\&A, 20, 249

Hartmann, L. 2002, ApJ, 566, L29 
Hasegawa, M., \& Nakazawa, K. 1990, A\&A, 227, 619

Hayashi, C. 1981, Prog. Theor. Phys. Suppl., 70, 35

Hedman, M. M., Nicholson, P. D., Salo, H., Wallis, B. D., Buratti, B. J., Baines, K. H., Brown, R. H., \& Clark, R. N. 2007, AJ, 133, 2624

Herbst, W., \& Mundt, R. 2005, ApJ, 633, 967

Ida, S. 1990, Icarus, 88, 129

Ida, S., \& Lin, D. N. C. 2004, ApJ, 604, 388

Ida, S., \& Lin, D. N. C. 2008, ApJ, 673, 487

Ida, S., \& Lin, D. N. C., submitted

Iess, L., Rappaport, N. J., Jacobson, R. A., Racioppa, P., Stevenson, D. J., Tortora, P., Armstrong, J. W., \& Asmar, S. W. 2010, Science, 327, 1367

Ikoma, M., Nakazawa, K., \& Emori, H. 2000, ApJ, 537, 1013

Kokubo, E., \& Ida, S. 1998, Icarus 131, 171

Königl, A. 1991, ApJ, 370, L39

Lin, D. N. C., \& Papaloizou, J. C. B. 1985, in Protostars and Planets II, ed. D. C. Black, \& M. S. Matthews (Tucson: Univ. Arizona Press), 981

Lubow, S. H., \& D'Angelo, G. 2006, ApJ, 641, 526

Lubow, S. H., Seibert, M., \& Artymowicz, P. 1999, ApJ, 526, 1001

Machida, M. N., Kokubo, E., \& Inutsuka, S. 2008, ApJ, 685, 1220

Machida, M. N. 2009, MNRAS, 392, 514

Masset, F. S., Morbidelli, A. Crida, A., \& Ferreira, J. 2006, ApJ, 642, 478

Mayor, M., et al. 2009, A\&A, 493, 639

Meyer, M. R., Backman, D. E., Weinberger, A. J., \& Wyatt, M. C. 2007, in Protostars and Planets V, ed. B. Reipurth, D. Jewitt, \& K. Keil (Tucson: Univ. Arizona Press), 573

Mosqueira, I., \& Estrada, P. R. 2003a, Icarus, 163, 198

Mosqueira, I., \& Estrada, P. R. 2003b, Icarus, 163, 232

Mosqueira, I., Estrada, P. R., \& Charnoz, S. 2010, Icarus, doi:10.1016/j.icarus.2009.10.018 (in press) 
Ogihara, M., \& Ida, S. 2009, ApJ, 699, 824

Ogihara, M., Duncan, M. J., \& Ida, S. 2010, ApJ, submitted

Ohtsuki K., Stewart, G. R., \& Ida, S. 2002, Icarus, 155, 436

Paardekooper, S.-J., Baruteau, C., Crida, A., \& Kley, W. 2009, MNRAS, doi:10.1111/j.13652966.2009.15782.x

Peale, S. J., \& Lee, M. H. 2002, Science, 298, 593

Pollack, J. B., Hubickyj, O., Bodenheimer, P., Lissauer, J. J., Podolak, M., \& Greenzweig, Y. 1996, Icarus, 124,62

Rebull, L. M., Stauffer, J. R., Megeath, S. T., Hora, J. L., \& Hartmann, L. 2006, ApJ, 646, 297

Robbins, S. J., Stewart, G. R., Lewis, M. C., Colwell, J. E., \& Sremcevic, M. 2010, Icarus, 206, 431

Sano T., Inutsuka, S., Turner, N. J., \& Stone, J. M. 2004, ApJ, 605, 321

Schubert, G., Anderson, J. D., Spohn, T., \& McKinnon, W. B. 2004, in Jupiter: The Planet, Satellites and Magnetosphere, ed. F. Gagenal, T. Dowling, \& W. B. McKinnon (United Kingdom: Cambridge Univ. Press), 281

Shakura, N. I., \& Sunyaev, R. A. 1973, A\&A, 24, 337

Stevenson, D. J. 1974, Icarus, 22, 403

Stevenson, D. J., Harris, A. W., \& Lunine, J. I. 1986, in Satellites, ed. J. A. Burns \& M. S. Matthews (Tucson: Univ. Arizona Press), 39

Stewart, G. R., \& Ida, S. 2000, Icarus, 143, 28

Takata, T., \& Stevenson, D. J. 1996, Icarus, 123, 404

Tanaka, H., Takeuchi, T., \& Ward, W. R. 2002, ApJ, 565, 1257

Tanaka, H., \& Ward, W. R. 2004, ApJ, 602, 388

Tanigawa, T., \& Ikoma, M. 2007, ApJ, 641, 526

Tiscareno, M. S., Burns, J. A., Hedman, M. M., \& Porco, C. C. 2008, AJ, 135, 1083

Ward, W. R., 1986, Icarus, 67, 164 


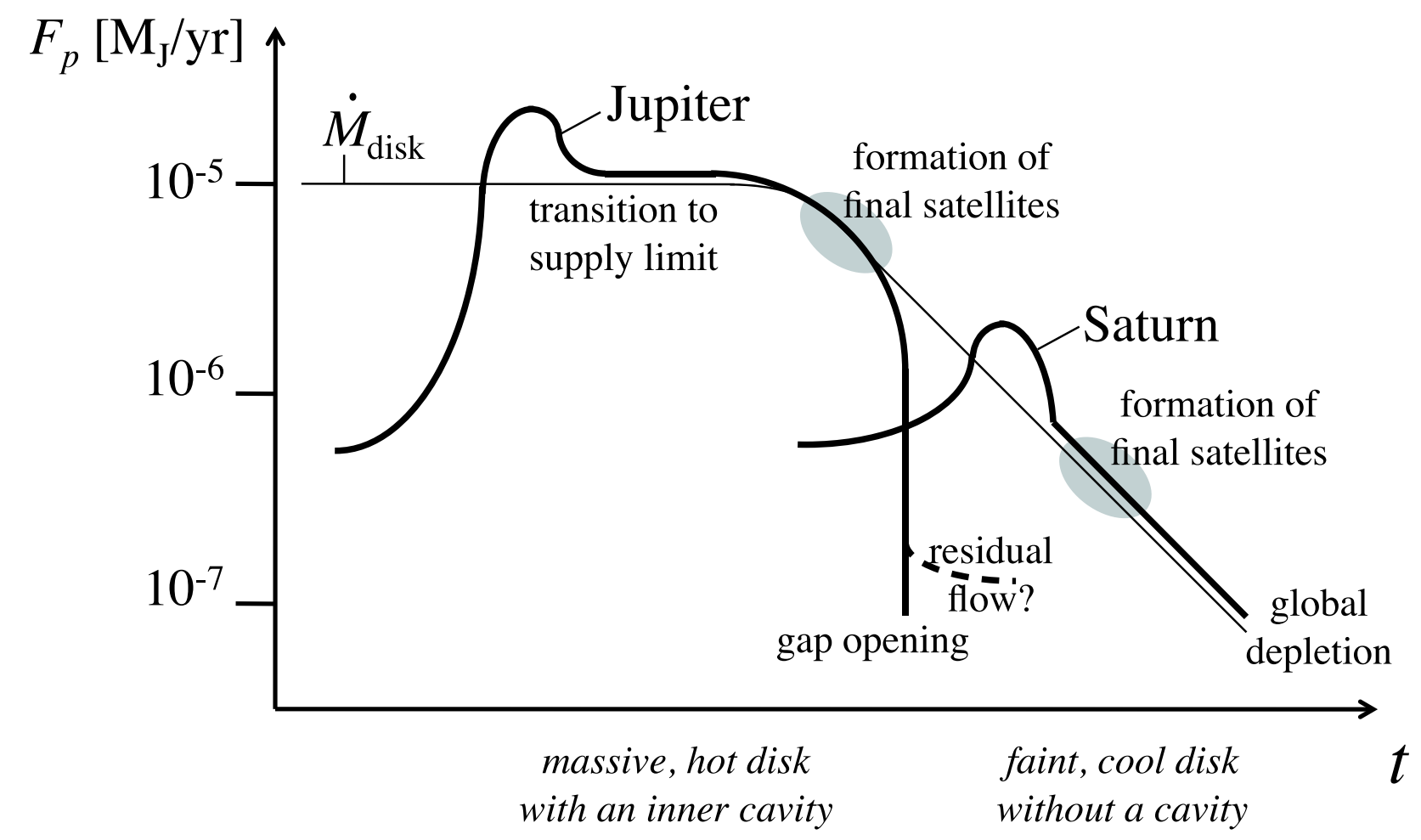

Fig. 1.- Schematic illustration for evolution of the infall rate $\left(F_{p}\right)$ onto the proto-satellite disks and timing of satellite formation in Jovian and Saturnian systems. Since $\Sigma_{g} \propto F_{p}^{3 / 4}$ and $T_{d} \propto F_{p}^{1 / 4}$, high $F_{p}$ means a massive and hot disk. 


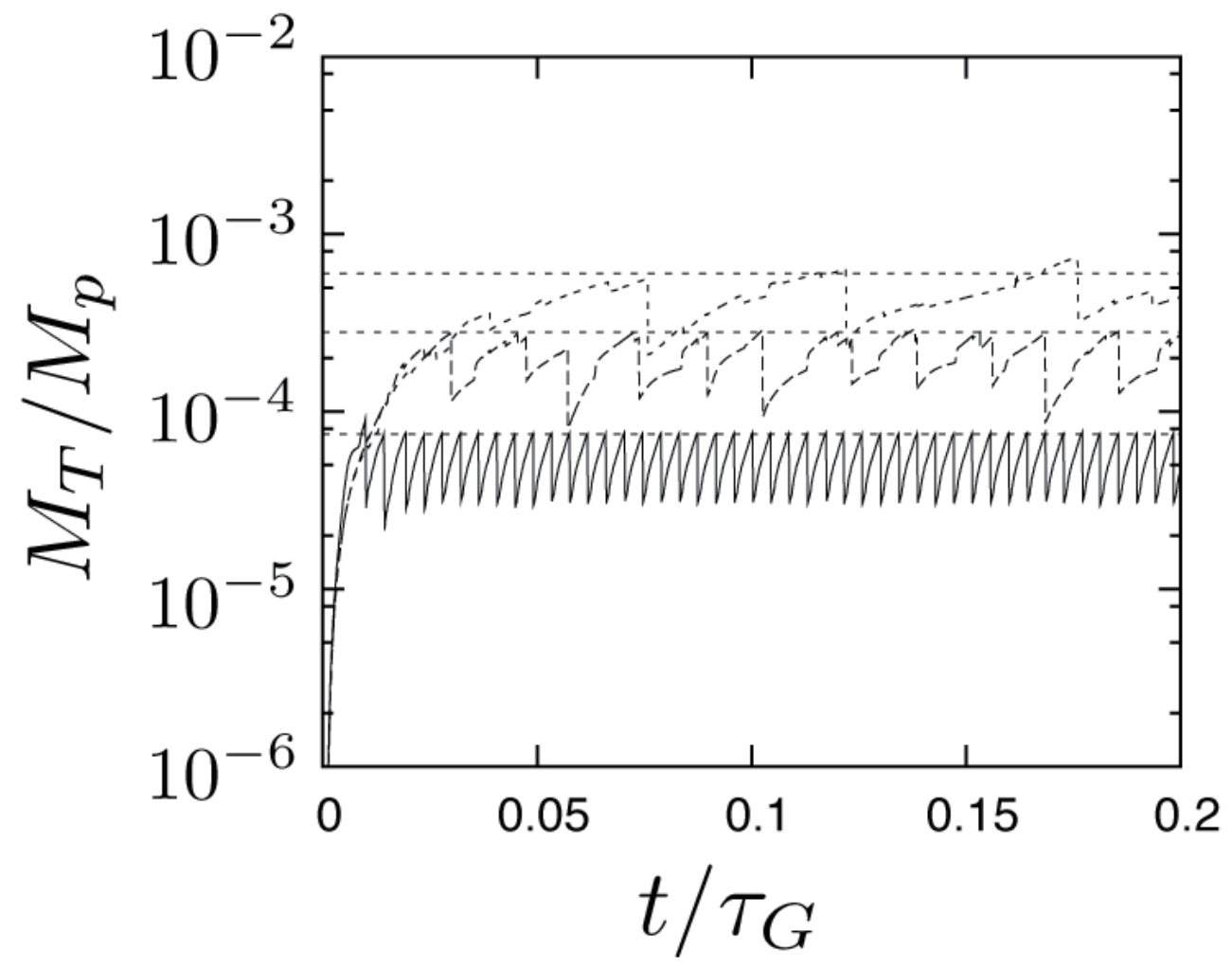

Fig. 2.- Results of calculations with time-constant inflows to be compared with Canup \& Ward (2006)'s N-body simulation (Fig. 2 in their paper). The non-cavity condition is adopted for a Jupiter mass planet. The total mass in surviving satellites, $M_{T}$, scaled by the planet's mass $\left(M_{p}\right)$ is shown versus time scaled to $\tau_{G}$. The solid, dashed and dotted lines correspond respectively to calculations with $\alpha=10^{-4}, 5 \times 10^{-3}$, and $5 \times 10^{-2}$. Dotted horizontal lines are predicted values of $M_{T} / M_{p}$ from Eq. (3) of Canup \& Ward (2006). 

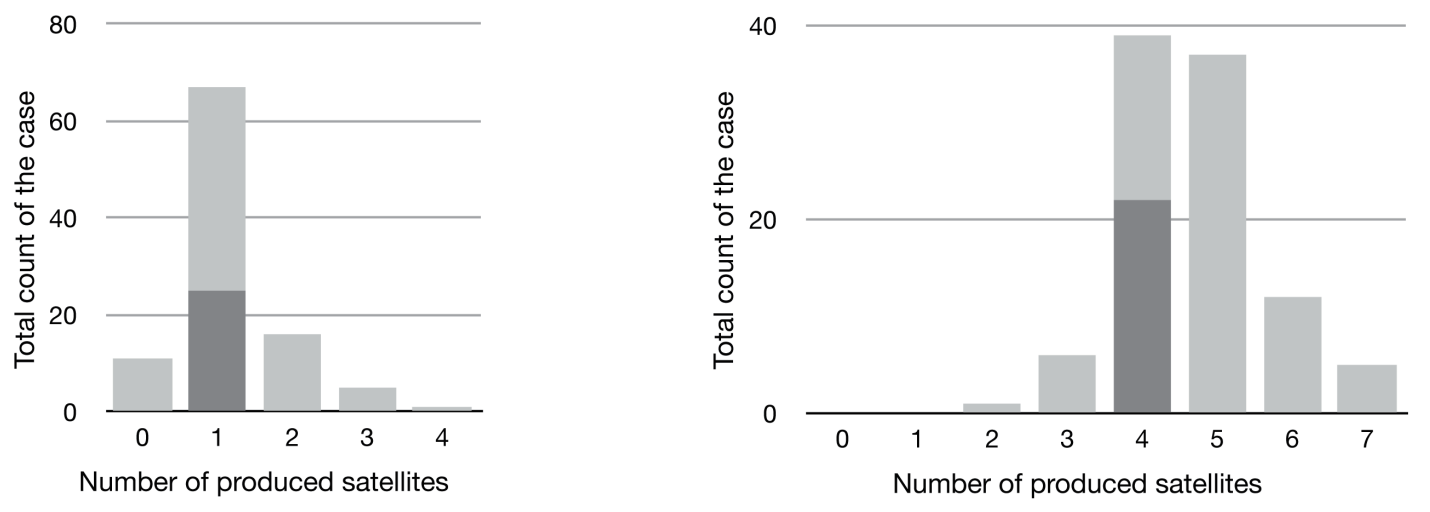

Fig. 3.- Distribution of the number of final surviving satellites with $M>10^{-5} M_{p}$ produced from 100 simulations for each system, (a) Saturnian system and (b) Jovian system. Dark gray parts show the runs that the produced satellite systems are analogous to the real one: for the Saturnian system, the largest satellite is icy and $M>10^{-4} M_{p}$, while for the Jovian system, the inner two bodies are rocky and the outer two are icy satellites.

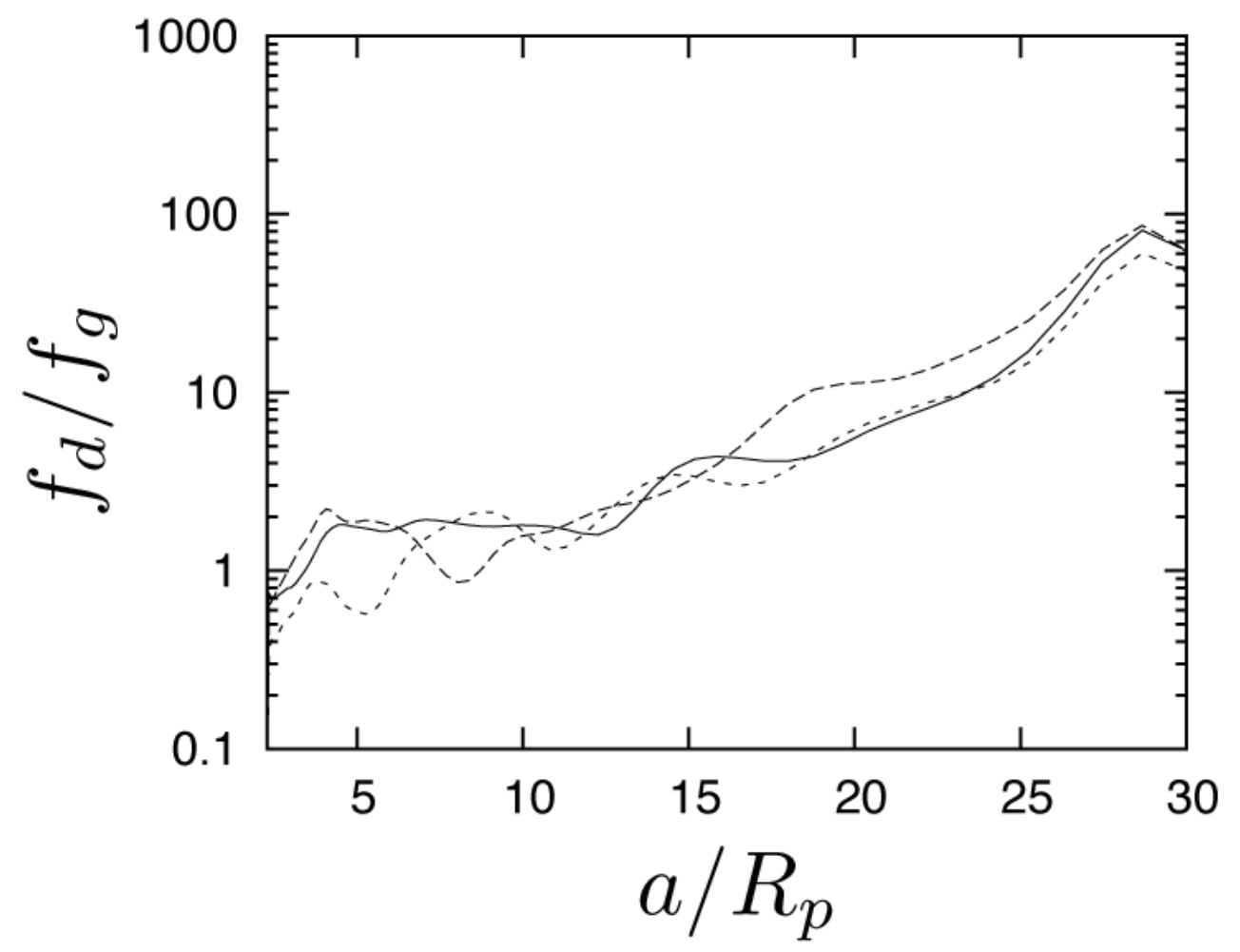

Fig. 4.- Dust to gas ratio in the steady state disk for three typical Saturnian cases. The solid, dashed and dotted lines correspond respectively to calculations with $N=15,18$, and 20 . If $f_{d} / f_{g}=1$, the ratio is the same as that of infalling gas, $1 / 100$. 

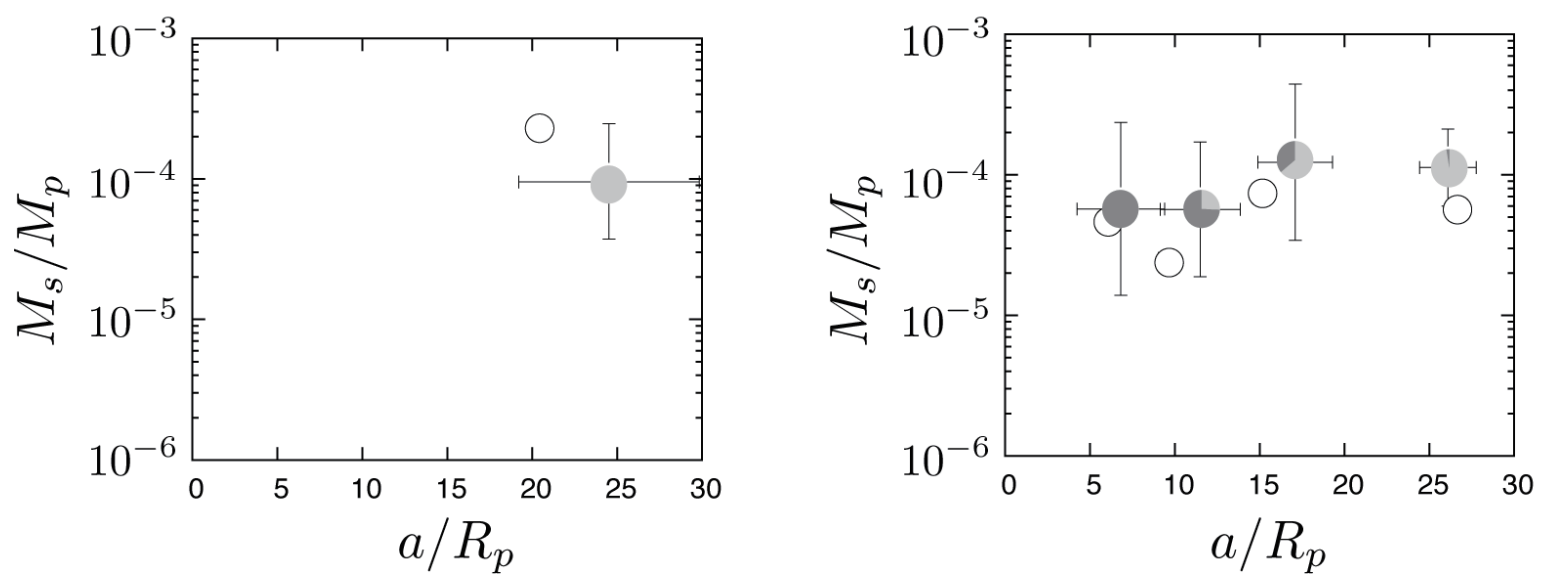

Fig. 5. - Theoretically predicted satellite systems in (a) set II corresponding to Saturnian systems with the data of Titan and (b) set I for Jovian systems with the data of Galilean satellites. For the Saturnian case, the average mass $\left(M_{s}\right)$ and semimajor axis $(a)$ of the largest satellites with their standard deviations are plotted with filled circles with bars for the highest probability cases (67 runs among 100 runs) in which only one large satellite $\left(M_{s}>10^{-5} M_{p}\right)$ is produced. The open circle represents Titan. For the Jovian case, the averaged mass-semimajor axis and their standard deviations from the highest probability runs (39 runs) that produced four large satellites are plotted. Galilean satellites are represented by open circles. The colors of each plot show the average fraction of rocky (dark gray) and icy (light gray) components of the formed satellites.
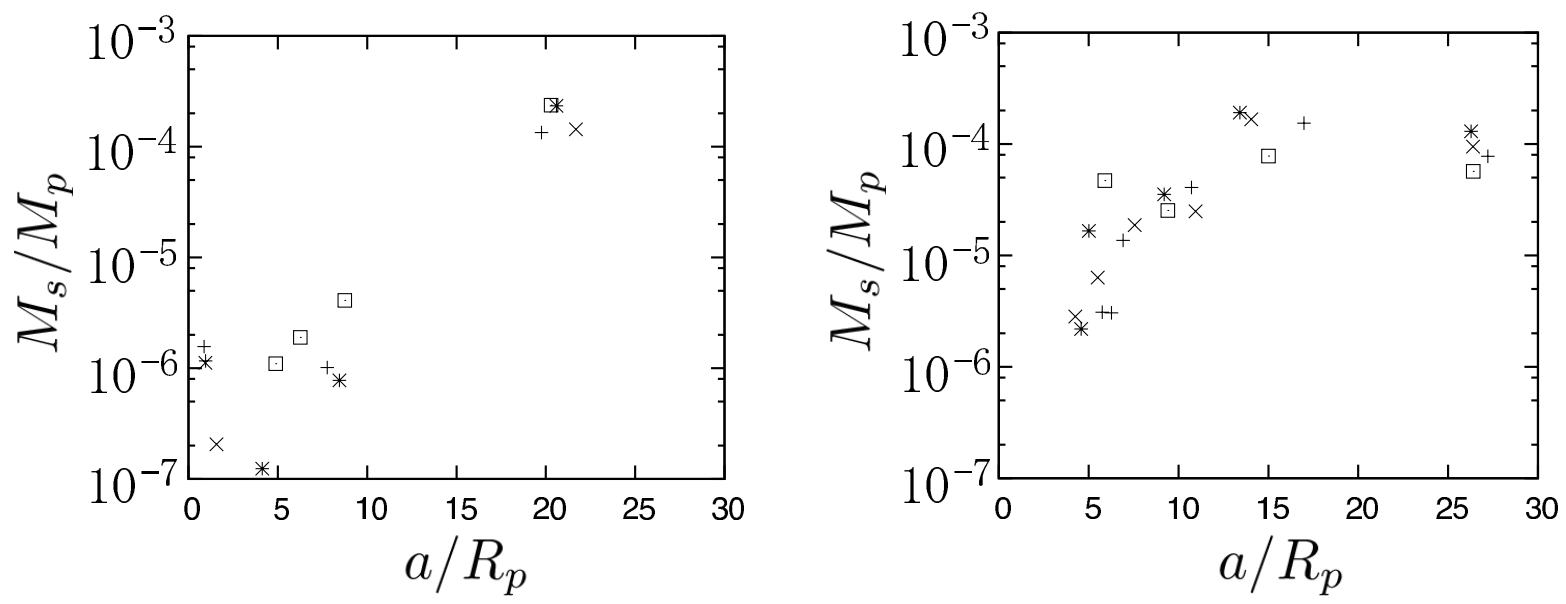

Fig. 6. - Final satellite systems for successful Saturnian and Jovian-like cases in (a) set II corresponding to Saturnian systems with the data of large satellites (Tethys, Dione, Dione, and Titan) and (b) set I for Jovian systems with the data of Galilean satellites. The mass $\left(M_{s}\right)$ and semimajor axis $(a)$ of the produced satellites are plotted with crosses, pluses, and stars for each successful case, while the open squares represent observed satellite systems. 
Table 1: Satellite properties* .

\begin{tabular}{lcccc}
\hline \hline Satellite & $a / R_{p}$ & $M / M_{p}\left[10^{-5}\right]$ & $\rho\left[\mathrm{g} \mathrm{cm}^{-3}\right]$ & $C / M R^{2}$ \\
\hline Io & 5.9 & 4.70 & 3.53 & 0.378 \\
Europa & 9.4 & 2.53 & 2.99 & 0.346 \\
Ganymede & 15.0 & 7.80 & 1.94 & 0.312 \\
Callisto & 26.4 & 5.69 & 1.83 & 0.355 \\
Titan & 20.3 & 23.7 & 1.88 & 0.34 \\
\hline
\end{tabular}

*Source: Schubert et al. 2004); Iess. et al. (2010) 
Table 2: Simulation settings for Jovian (set I) and Saturnian (set II) systems.

\begin{tabular}{l|lccc}
\hline & $F_{p, 0}$ & inner cavity & disk depletion & $\alpha$ \\
\hline \hline Jovian systems (set I) & $M_{p} /\left(5 \times 10^{6}\right.$ yrs $)$ & Yes & a & $10^{-3}-10^{-2 *}$ \\
\hline Jovian systems (set I') & $M_{p} /\left(5 \times 10^{6}\right.$ yrs $)$ & Yes & b & $10^{-3}-10^{-2}$ \\
\hline Saturnian systems (set II) & $M_{p} /\left(2 \times 10^{6}\right.$ yrs $)$ & No & c & $10^{-3}-10^{-2}$ \\
\hline
\end{tabular}

a) The evolution of $F_{p}$ is the same as c) until it is truncated at $t=(0.5-0.8) \tau_{\text {dep }}$, which is corresponding to the gap formation.

b) The same as a) except that $F_{p}$ is reduced by a factor of 100 instead of complete vanishment at the gap formation.

c) After establishment of steady state, the infall is decayed as $F_{p}=F_{p, 0} \exp \left(-t / \tau_{\text {dep }}\right)$ with $\tau_{\text {dep }}=(3-5) \times 10^{6}$ yrs. *

*a $\log$ uniform distribution 